\title{
HST-COS OBSERVATIONS OF AGNs. II. EXTENDED SURVEY OF ULTRAVIOLET COMPOSITE SPECTRA FROM 159 ACTIVE GALACTIC NUCLEI*
}

\author{
Matthew L. Stevans ${ }^{1}$, J. Michael Shull ${ }^{2}$, Charles W. Danforth, and Evan M. Tilton \\ CASA, Department of Astrophysical and Planetary Sciences, University of Colorado, Boulder, CO 80309, USA; stevans@astro.as.utexas.edu, \\ michael.shull@colorado.edu, charles.danforth@colorado.edu, evan.tilton@colorado.edu \\ Received 2014 June 25; accepted 2014 August 19; published 2014 September 24
}

\begin{abstract}
The ionizing fluxes from quasars and other active galactic nuclei (AGNs) are critical for interpreting their emissionline spectra and for photoionizing and heating the intergalactic medium. Using far-ultraviolet (FUV) spectra from the Cosmic Origins Spectrograph (COS) on the Hubble Space Telescope (HST), we directly measure the rest-frame ionizing continua and emission lines for 159 AGNs at redshifts $0.001<z_{\mathrm{AGN}}<1.476$ and construct a composite spectrum from 475 to $1875 \AA$. We identify the underlying AGN continuum and strong extreme ultraviolet (EUV) emission lines from ions of oxygen, neon, and nitrogen after masking out absorption lines from the H I Ly $\alpha$ forest, 7 Lyman-limit systems $\left(N_{\mathrm{H}_{\mathrm{I}}} \geqslant 10^{17.2} \mathrm{~cm}^{-2}\right)$ and 214 partial Lyman-limit systems $\left(14.5<\log N_{\mathrm{H}_{\mathrm{I}}}<17.2\right)$. The 159 AGNs exhibit a wide range of FUV/EUV spectral shapes, $F_{v} \propto v^{\alpha_{v}}$, typically with $-2 \leqslant \alpha_{\nu} \leqslant 0$ and no discernible continuum edges at $912 \AA$ (H I) or $504 \AA$ (He I). The composite rest-frame continuum shows a gradual break at $\lambda_{\text {br }} \approx 1000 \AA$, with mean spectral index $\alpha_{v}=-0.83 \pm 0.09$ in the FUV (1200-2000 $)$ steepening to $\alpha_{v}=-1.41 \pm 0.15$ in the EUV (500-1000 $\AA$ ). We discuss the implications of the UV flux turnovers and lack of continuum edges for the structure of accretion disks, AGN mass inflow rates, and luminosities relative to Eddington values.
\end{abstract}

Key words: galaxies: active - line: profiles - quasars: emission lines - ultraviolet: galaxies

Online-only material: color figures

\section{INTRODUCTION}

The far ultraviolet (FUV) and extreme ultraviolet (EUV) continua of active galactic nuclei (AGNs) are thought to form in the black hole accretion disk (Krolik 1999; Koratkar \& Blaes 1999), but their ionizing photons can influence physical conditions in the broad emission-line region of the AGN as well as surrounding interstellar and intergalactic gas. The metagalactic background from galaxies and AGNs is also an important parameter in cosmological simulations as a dominant source of ionizing radiation, critical for interpreting broad emission-line spectra of AGNs, intergalactic metal-line absorbers, and fluctuations in the ratio of the Ly $\alpha$ absorbers of H I and He II.

Since the deployment of the first space-borne ultraviolet (UV) spectrographs, astronomers have combined spectral observations of AGNs into composite spectra. These composites constrain the intensity and shape of the AGN component of the ionizing photon background. The most direct probe of the FUV and EUV continua in the AGN rest frame comes from observations taken by instruments such as the International Ultraviolet Explorer (IUE) and a series of UV spectrographs on board the Hubble Space Telescope (HST): the Goddard High Resolution Spectrograph (GHRS), the Faint Object Spectrograph (FOS), the Space Telescope Imaging Spectrograph (STIS), and the Cosmic Origins Spectrograph (COS). Ultraviolet (UV) spectra were also obtained by the Far Ultraviolet Spectroscopic Explorer (FUSE). For AGNs at modest redshifts, all of these

\footnotetext{
* Based on observations made with the NASA/ESA Hubble Space Telescope, obtained from the data archive at the Space Telescope Science Institute. STScI is operated by the Association of Universities for Research in Astronomy, Inc. under NASA contract NAS5-26555.

1 Present address: Astronomy Department, University of Texas, Austin, TX 78712, USA.

2 Also at Institute of Astronomy, Cambridge University, Cambridge CB3 OHA, UK.
}

instruments provide access to the rest-frame Lyman continuum (LyC, $\lambda<912 \AA$ ) and at $z<1.5$ they avoid strong contamination from the Ly $\alpha$-forest absorbers in the spectra of highredshift AGNs. Thus, obtaining access to high signal-to-noise $(\mathrm{S} / \mathrm{N})$, moderate-resolution UV spectra is crucial for finding a reliable underlying continuum.

This is our second paper, following an AGN composite spectrum presented in Shull et al. (2012, hereafter Paper I) based on initial results from COS spectra of 22 AGNs at redshifts $0<$ $z<1.4$. We analyzed their rest-frame FUV and EUV spectra, taken with the G130M and G160M gratings, whose resolving power $R=\lambda / \Delta \lambda \approx 18,000\left(17 \mathrm{~km} \mathrm{~s}^{-1}\right.$ velocity resolution $)$ allows us to distinguish line blanketing from narrow interstellar and intergalactic absorption lines. Here in Paper II, we enlarge our composite spectrum from 22 to 159 AGNs, confirm the validity of our early results, and explore possible variations of the EUV spectral index with AGN type and luminosity. Both studies were enabled by high-quality, moderate-resolution spectra taken with the Cosmic Origins Spectrograph installed on the HST during the 2009 May servicing mission. The COS instrument (Green et al. 2012) was designed explicitly for point-source spectroscopy of faint targets, particularly quasars and other AGNs used as background sources for absorptionline studies of the intergalactic medium (IGM), circumgalactic medium (CGM), and interstellar medium (ISM). Our survey is based on high-quality spectra of the numerous AGNs used in these projects.

Our expanded survey of 159 AGNs finds a composite spectral energy distribution (SED) with frequency index $\alpha_{v}=-1.41 \pm$ 0.15 in the rest-frame EUV. This confirms the results of Paper I, where we found $\alpha_{\nu}=-1.41 \pm 0.21$. We adopt the convention in which rest-frame flux distributions are fitted to power laws in wavelength, $F_{\lambda} \propto \lambda^{\alpha_{\lambda}}$, and converted to $F_{v} \propto \nu^{\alpha_{\nu}}$ in frequency with $\alpha_{\nu}=-\left(2+\alpha_{\lambda}\right)$. We caution that these spectral 
indices are local measures of the slope over a small range in wavelength, $\Delta \lambda / \lambda \approx 0.45$. Because of the curvature of the AGN spectral distributions, local slopes can be misleading when compared to different wavelength bands and to objects at different redshift. We return to this issue in Section 3.3, where we discuss possible correlations of indices $\alpha_{\lambda}$ with AGN type, redshift, and luminosity and compare indices measured by both HST/COS and FUSE.

The COS composite spectrum is somewhat harder than that in earlier HST/FOS and STIS observations by Telfer et al. (2002), who fitted the continuum (500-1200 А) with $\alpha_{v}=$ $-1.76 \pm 0.12$ for 184 QSOs at $z>0.33$. Their sample of 39 radio-quiet AGNs had $\alpha_{v}=-1.57 \pm 0.17$. Our fit differs considerably from the FUSE survey of 85 AGNs at $z \leqslant 0.67$ by Scott et al. (2004), who found a harder composite spectrum with $\alpha_{v}=-0.56_{-0.28}^{+0.38}$. The different indices could arise in part from the small numbers of targets observed in the rest-frame EUV. Even in the current sample, only 10 or fewer AGN observations cover the spectral range $450 \AA \lesssim \lambda \lesssim 600 \AA$. Another important difference in methodology is our placement of the EUV continuum relative to strong emission lines such as Ne VIII (770, $780 \AA), \operatorname{Ne~v~(570~\AA ),~O~II~(834~})$, O III (833, $702 \AA)$, O IV (788, $554 \AA)$, O v (630 ̊), and O vi (1032, $1038 \AA)$. Identifying and fitting these emission lines requires high $\mathrm{S} / \mathrm{N}$ spectra. A complete list of lines appears in Table 4 of Paper I. We also use the higher spectral resolution of the COS (G130M and G160M) gratings to distinguish the line blanketing by narrow absorption lines from the Ly $\alpha$ forest. Increasingly important at higher redshifts, we need to identify and correct for absorption from Lyman-limit systems (LLS) with $N_{\mathrm{HI}} \gtrsim 10^{17.2} \mathrm{~cm}^{-2}$ and partial Lyman-limit systems (pLLS) with $N_{\mathrm{HI}}=10^{15}-10^{17.2} \mathrm{~cm}^{-2}$. The historical boundary at $10^{17.2} \mathrm{~cm}^{-2}$ occurs where the photoelectric optical depth $\tau_{\mathrm{HI}}=1$ at the $912 \AA$ Lyman edge.

In Paper I, our 22 AGNs ranged in redshift from $z=0.026$ to $z=1.44$, but included only four targets at sufficient redshift to probe the rest-frame continuum below $550 \AA$. Our new survey contains 159 AGNs out to $z=1.476$ with 16 targets at $z>0.90$, sufficient to probe below $600 \AA$ by observing with G130M down to $1135 \AA$. In all AGN spectra, we identify the prominent broad emission lines and line-free portions of the spectrum and fit the underlying continua, excluding interstellar and intergalactic absorption lines. In Section 2, we describe the $\operatorname{COS}$ data reduction and our new techniques for restoring the continua with a fitting method that corrects for the effects of absorption from the IGM and ISM. In Section 3, we describe our results on the FUV and EUV spectral indices in both individual and composite spectra. Section 4 presents our conclusions and their implications.

\section{OBSERVATIONS OF ULTRAVIOLET SPECTRA OF AGNS}

\subsection{Sample Description}

Table 1 lists the relevant COS observational parameters of our 159 AGN targets, which include AGN type and redshift $\left(z_{\mathrm{AGN}}\right)$, from $\mathrm{NED},{ }^{3}$ continuum index $\left(\alpha_{\lambda}\right)$, fitted rest-frame flux normalization at $1100 \AA$, observed flux $F_{\lambda}$ at $1300 \AA$, and $\mathrm{S} / \mathrm{N}$ ratios. We also provide power-law fits to their $H S T / \mathrm{COS}$

\footnotetext{
3 NASA/IPAC Extragalactic Database (NED) is operated by the Jet Propulsion Laboratory, California Institute of Technology, under contract with the National Aeronautics and Space Administration, http://nedwww.ipac.caltech.edu.
}

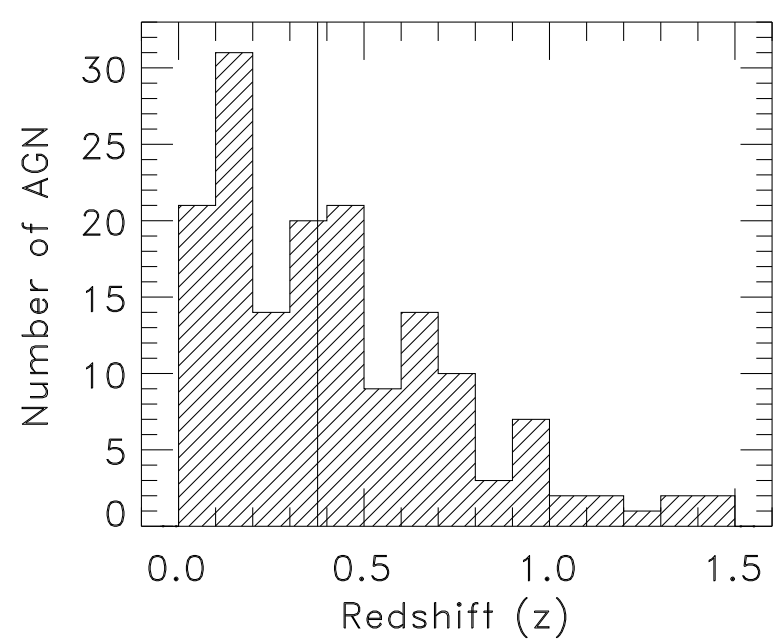

Figure 1. Histogram of the redshifts of the 159 AGNs in our COS sample. Vertical line marks the median redshift, $\langle z\rangle \approx 0.37$ of the sample.

spectra (see Section 2.2) and their monochromatic luminosities, $\lambda L_{\lambda}$, at $1100 \AA$, given by

$\lambda L_{\lambda}=\left(1.32 \times 10^{43} \mathrm{ergs}^{-1}\right)\left[\frac{d_{L}}{100 \mathrm{Mpc}}\right]^{2}\left[\frac{F_{\lambda}}{10^{-14}}\right]\left[\frac{\lambda}{1100 \AA}\right]$.

We converted flux, $F_{\lambda}$ (in $\operatorname{erg} \mathrm{cm}^{-2} \mathrm{~s}^{-1} \AA^{-1}$ ) to monochromatic luminosity, $L_{\lambda}=4 \pi d_{L}^{2} F_{\lambda}$, using the luminosity distance, $d_{L}(z)$, computed for a flat $\Lambda \mathrm{CDM}$ universe with $H_{0}=$ $70 \mathrm{~km} \mathrm{~s}^{-1} \mathrm{Mpc}^{-1}$ and density parameters $\Omega_{m}=0.275$ and $\Omega_{\Lambda}=0.725$ (Komatsu et al. 2011).

The redshift distribution of the sample is shown in Figure 1, and the target distribution in accessible rest-frame wavelength in Figure 2. Our sample consists only of those AGNs observed with both the G130M (1135-1460 ̊) and G160M (1390-1795 A) COS gratings, providing the broad wavelength coverage at $17 \mathrm{~km} \mathrm{~s}^{-1}$ resolution needed for our study of the continuum, emission lines, and absorption line blanketing. The COS instrument and data acquisition are described by Osterman et al. (2011) and Green et al. (2012). We retrieved all COS AGN spectra publicly available as of 2013 April 25, but excluded spectra with low $\mathrm{S} / \mathrm{N}$ per pixel $(\mathrm{S} / \mathrm{N}<1)$ and all BL Lac objects, which are over-represented in the COS archives. We also excluded three targets with abnormal spectra that would complicate the analysis: SDSSJ004222.29-103743.8, which exhibits broad absorption lines; SDSSJ135726.27+043541.4, which features a pLLS longward of the COS waveband; and UGCA 166, which Gil de Paz \& Madore (2005) classify as a blue compact dwarf galaxy. This leaves 159 AGNs for analysis.

\subsection{Data Acquisition and Processing}

We follow the same procedure as in Paper I for obtaining, reducing, and processing the data. Below, we briefly summarize the procedure and explicitly note improvements or deviations from earlier methods. Many of these techniques of coaddition and continuum fitting were also discussed in our IGM survey (Danforth et al. 2014). Of particular interest in this paper are new techniques for identifying LLS and pLLS absorbers, measuring their $\mathrm{H}$ I column densities, and using that information to correct the continuum. Our analysis proceeds through the following steps.

1. Retrieve exposures. The CALCOS calibrated exposures were downloaded from the Mikulski Archive for Space 
Table 1

COS Observations (159 AGN Targets Ordered by Redshift)

\begin{tabular}{|c|c|c|c|c|c|c|c|}
\hline $\begin{array}{l}\text { AGN }^{\mathrm{a}} \\
\text { Target }\end{array}$ & $\begin{array}{l}\text { AGN }^{a} \\
\text { Type }\end{array}$ & $z^{\mathrm{a}}$ & $\alpha_{\lambda}$ & $\begin{array}{c}F_{0}^{\mathrm{a}} \\
(1100 \AA)\end{array}$ & $\begin{array}{c}F_{\lambda}^{\mathrm{a}} \\
(1300 \AA)\end{array}$ & $\begin{array}{c}\log \left(\lambda L_{\lambda}\right)^{\mathrm{a}} \\
(1100 \AA)\end{array}$ & $(\mathrm{S} / \mathrm{N})_{\mathrm{res}}^{\mathrm{a}}$ \\
\hline NGC 4395 & Sy 1.8 & 0.001064 & $-0.62 \pm 0.006$ & 0.188 & 0.16 & 39.70 & 4,4 \\
\hline NGC 4051 & NLSy 1 & 0.002336 & $-0.07 \pm 0.002$ & 0.809 & 0.83 & 41.02 & 16,13 \\
\hline NGC 3516 & Sy 1.5 & 0.008836 & $-0.03 \pm 0.007$ & 1.54 & 1.1 & 42.46 & 14,12 \\
\hline NGC 3783 & Sy 1.5 & 0.00973 & $-1.59 \pm 0.1$ & 11.0 & 3.6 & 43.40 & 21,17 \\
\hline NGC 7469 & Sy 1.5 & 0.016317 & $-1.10 \pm 0.03$ & 11.9 & 6.4 & 43.88 & 28,18 \\
\hline Mrk1044 & NLSy 1 & 0.016451 & $-0.99 \pm 0.007$ & 3.98 & 2.6 & 43.41 & 19,13 \\
\hline NGC 5548 & Sy 1.5 & 0.017175 & $-1.62 \pm 0.003$ & 9.56 & 7.0 & 43.83 & 28,19 \\
\hline AKN564 & LINER & 0.024684 & $-0.94 \pm 0.02$ & 1.20 & 0.67 & 43.25 & 9,7 \\
\hline Mrk335 & Sy 1 & 0.025785 & $-1.51 \pm 0.009$ & 5.82 & 4.1 & 43.98 & 24,18 \\
\hline ESO031-G008 & Sy 1.2 & 0.027619 & $-1.79 \pm 0.01$ & 0.958 & 0.62 & 43.25 & 9,7 \\
\hline Mrk290 & Sy 1.5 & 0.029577 & $-0.78 \pm 0.003$ & 1.80 & 1.8 & 43.59 & 20,16 \\
\hline Mrk279 & Sy 1 & 0.030451 & $-0.61 \pm 0.003$ & 0.419 & 0.49 & 42.98 & 8,7 \\
\hline Mrk817 & Sy 1.5 & 0.031455 & $-1.27 \pm 0.0003$ & 8.92 & 9.4 & 44.34 & 38,24 \\
\hline Mrk509 & Sy 1.5 & 0.034397 & $-1.58 \pm 0.02$ & 17.8 & 12.6 & 44.72 & 63,55 \\
\hline PG1011-040 & Sy 1.2 & 0.058314 & $-1.75 \pm 0.01$ & 2.63 & 2.7 & 44.36 & 26,13 \\
\hline Mrk1513 & Sy 1.5 & 0.062977 & $-1.41 \pm 0.02$ & 2.55 & 5.7 & 44.42 & 27,15 \\
\hline MR2251-178 & Sy 1.5 & 0.06398 & $-1.04 \pm 0.009$ & 4.26 & 25.3 & 44.65 & 33,25 \\
\hline RXJ0503.1-6634 & Sy 1 & 0.064 & $-2.06 \pm 0.05$ & 1.30 & 2.2 & 44.14 & 16,8 \\
\hline SDSSJ145108.76+270926.9 & NLSy 1 & 0.0645 & $-0.81 \pm 0.008$ & 0.539 & 0.92 & 43.77 & 10,8 \\
\hline RBS563 & Sy 1.5 & 0.069 & $-1.50 \pm 0.005$ & 0.574 & 9.0 & 43.85 & 13,7 \\
\hline SDSSJ031027.82-004950.7 & Sy 1 & 0.080139 & $-1.29 \pm 0.03$ & 0.827 & 1.0 & 44.15 & 10,8 \\
\hline PG0804+761 & Sy 1 & 0.101 & $-1.46 \pm 0.007$ & 11.0 & 9.6 & 45.48 & 51,33 \\
\hline IRAS-F22456-5125 & Sy 1.5 & 0.101 & $-1.76 \pm 0.0006$ & 2.18 & 2.5 & 44.77 & 44,24 \\
\hline UKS-0242-724 & Sy 1.2 & 0.1018 & $-1.66 \pm 0.007$ & 1.34 & 1.1 & 44.58 & 13,9 \\
\hline IRAS-F04250-5718 & Sy 1.5 & 0.104 & $-1.72 \pm 0.001$ & 3.76 & 3.7 & 45.05 & 63,34 \\
\hline TonS210 & Sy 1 & 0.116 & $-1.74 \pm 0.002$ & 4.18 & 3.7 & 45.19 & 36,21 \\
\hline Q1230+0115 & NLSy 1 & 0.117 & $-0.34 \pm 0.002$ & 3.33 & 4.2 & 45.10 & 51,31 \\
\hline HS0033+4300 & Sy 1 & 0.12 & $-0.93 \pm 0.02$ & 0.241 & 0.12 & 43.99 & 6,7 \\
\hline Mrk106 & Sy 1 & 0.122951 & $-1.75 \pm 0.004$ & 1.44 & 1.1 & 44.79 & 23,14 \\
\hline SDSSJ152139.66+033729.2 & Sy 1 & 0.126354 & $-0.09 \pm 0.01$ & 0.192 & 0.17 & 43.94 & 5,4 \\
\hline Mrk876 & Sy 1 & 0.129 & $-1.18 \pm 0.004$ & 4.56 & 3.8 & 45.33 & 56,31 \\
\hline IRASL06229-6434 & Sy1 & 0.129 & $-0.83 \pm 0.02$ & 1.03 & 0.70 & 44.68 & 16,9 \\
\hline PG0838+770 & Sy 1 & 0.131 & $-1.12 \pm 0.005$ & 1.05 & 0.83 & 44.71 & 24,11 \\
\hline PG1626+554 & Sy 1 & 0.133 & $-1.88 \pm 0.0005$ & 2.41 & 2.2 & 45.08 & 24,14 \\
\hline QSO0045+3926 & Sy 1 & 0.134 & $-0.77 \pm 0.02$ & 1.22 & 0.77 & 44.79 & 29,20 \\
\hline PKS0558-504 & NLSy 1 & 0.1372 & $-1.75 \pm 0.02$ & 3.94 & 2.8 & 45.33 & 15,7 \\
\hline SDSSJ094733.21+100508.7 & Sy 1.5 & 0.139297 & $-1.91 \pm 0.004$ & 0.868 & 0.65 & 44.68 & 11,6 \\
\hline SDSSJ135712.61+170444.1 & QSO & 0.1505 & $-1.58 \pm 0.005$ & 0.584 & 0.44 & 44.58 & 12,8 \\
\hline SDSSJ112114.22+032546.7 & NLSy 1 & 0.152033 & $-0.17 \pm 0.02$ & 0.15 & 0.10 & 44.01 & 4,3 \\
\hline PG1115+407 & Sy 1 & 0.154567 & $-0.44 \pm 0.002$ & 1.04 & 1.0 & 44.86 & 19,11 \\
\hline SDSSJ095915.65+050355.1 & QSO & 0.162296 & $-1.56 \pm 0.006$ & 0.674 & 0.56 & 44.72 & 11,7 \\
\hline SDSSJ135625.55+251523.7 & Sy1 & 0.164009 & $-1.35 \pm 0.004$ & 0.464 & 0.39 & 44.57 & 8,5 \\
\hline SDSSJ015530.02-085704.0 & Sy 1 & 0.164427 & $-1.10 \pm 0.004$ & 0.4 & 0.35 & 44.51 & 9,6 \\
\hline PG1202+281 & Sy 1.2 & 0.1653 & $-0.46 \pm 0.005$ & 0.186 & 0.16 & 44.18 & 7,5 \\
\hline PG1048+342 & Sy 1 & 0.167132 & $-1.20 \pm 0.003$ & 0.657 & 0.59 & 44.74 & 18,12 \\
\hline SDSSJ121114.56+365739.5 & Sy 1 & 0.170796 & $-1.09 \pm 0.003$ & 0.502 & 0.44 & 44.64 & 10,7 \\
\hline SDSSJ134231.22+382903.4 & Sy 1 & 0.171869 & $-1.76 \pm 0.002$ & 0.488 & 0.49 & 44.63 & 10,6 \\
\hline 1SAXJ1032.3+5051 & QSO & 0.173128 & $-1.05 \pm 0.007$ & 0.095 & 0.08 & 43.93 & 9,5 \\
\hline SDSSJ021218.32-073719.8 & Sy 1 & 0.17392 & $-1.37 \pm 0.006$ & 0.251 & 0.21 & 44.36 & 8,5 \\
\hline PG1116+215 & Sy 1 & 0.1763 & $-1.43 \pm 0.003$ & 5.04 & 4.4 & 45.67 & 34,22 \\
\hline 2MASX-J01013113+4229356 & Sy 1 & 0.19 & $-1.04 \pm 0.05$ & 0.71 & 0.37 & 44.89 & 8,6 \\
\hline PHL1811 & NLSy 1 & 0.192 & $-1.93 \pm 0.02$ & 7.95 & 5.5 & 45.95 & 34,18 \\
\hline SDSSJ123604.02+264135.9 & QSO & 0.208995 & $-1.17 \pm 0.01$ & 0.165 & 0.19 & 44.35 & 7,5 \\
\hline PG1121+422 & Sy 1 & 0.225025 & $-1.43 \pm 0.005$ & 0.831 & 0.88 & 45.13 & 16,10 \\
\hline SDSSJ001224.01-102226.5 & QSO & 0.228191 & $-0.57 \pm 0.006$ & 0.283 & 0.21 & 44.67 & 7,5 \\
\hline PG0953+414 & Sy 1 & 0.2341 & $-2.04 \pm 0.001$ & 3.80 & 4.3 & 45.83 & 33,20 \\
\hline SDSSJ092909.79+464424.0 & QSO & 0.239959 & $-1.56 \pm 0.002$ & 1.24 & 1.3 & 45.37 & 14,9 \\
\hline SDSSJ133053.27+311930.5 & Sy 1.5 & 0.242204 & $-0.97 \pm 0.002$ & 0.308 & 0.44 & 44.77 & 10,7 \\
\hline RXJ0439.6-5311 & Sy 1 & 0.243 & $-1.70 \pm 0.002$ & 0.337 & 0.36 & 44.81 & 14,8 \\
\hline FBQSJ1010+3003 & QSO & 0.255778 & $-0.01 \pm 0.004$ & 0.224 & 0.38 & 44.68 & 15,9 \\
\hline SDSSJ115758.72-002220.8 & Sy 1 & 0.260247 & $-0.96 \pm 0.02$ & 0.274 & 0.57 & 44.79 & 8,5 \\
\hline SDSSJ134206.56+050523.8 & Sy 1.2 & 0.266015 & $-1.58 \pm 0.007$ & 0.446 & 0.81 & 45.02 & 10,7 \\
\hline PKS1302-102 & Sy 1.2 & 0.2784 & $-1.11 \pm 0.01$ & 1.79 & 1.6 & 45.67 & 23,16 \\
\hline Ton580 & Sy 1 & 0.290237 & $-0.43 \pm 0.002$ & 0.974 & 0.97 & 45.45 & 18,12 \\
\hline SDSSJ092837.98+602521.0 & Sy 1 & 0.29545 & $+0.76 \pm 0.005$ & 0.182 & 0.21 & 44.74 & 5,5 \\
\hline
\end{tabular}


Table 1

(Continued)

\begin{tabular}{|c|c|c|c|c|c|c|c|}
\hline $\begin{array}{l}\text { AGN }^{\mathrm{a}} \\
\text { Target }\end{array}$ & $\begin{array}{l}\mathrm{AGN}^{\mathrm{a}} \\
\text { Type }\end{array}$ & $z^{\mathrm{a}}$ & $\alpha_{\lambda}$ & $\begin{array}{c}F_{0}^{\mathrm{a}} \\
(1100 \AA ̊)\end{array}$ & $\begin{array}{c}F_{\lambda}^{\mathrm{a}} \\
(1300 \AA)\end{array}$ & $\begin{array}{c}\log \left(\lambda L_{\lambda}\right)^{\mathrm{a}} \\
(1100 \AA)\end{array}$ & $(\mathrm{S} / \mathrm{N})_{\mathrm{res}}^{\mathrm{a}}$ \\
\hline $\mathrm{H} 1821+643$ & Sy 1.2 & 0.2968 & $-0.52 \pm 0.007$ & 4.16 & 3.4 & 46.10 & 52,7 \\
\hline SDSSJ091235.42+295725.4 & QSO & 0.305331 & $-1.63 \pm 0.004$ & 0.098 & 0.13 & 44.50 & 6,5 \\
\hline SDSSJ082633.51+074248.3 & QSO & 0.310643 & $-1.12 \pm 0.009$ & 0.491 & 0.52 & 45.22 & 9,6 \\
\hline SDSSJ120720.99+262429.1 & QSO & 0.323529 & $-0.41 \pm 0.003$ & 0.387 & 0.40 & 45.16 & 8,7 \\
\hline SDSSJ134251.60-005345.3 & Sy 1 & 0.325 & $-1.34 \pm 0.005$ & 0.535 & 0.62 & 45.30 & 10,7 \\
\hline SDSSJ092554.43+453544.4 & QSO & 0.329478 & $-1.69 \pm 0.008$ & 0.429 & 0.63 & 45.22 & 13,11 \\
\hline PG1001+291 & Sy 1 & 0.3297 & $-1.30 \pm 0.003$ & 0.999 & 1.1 & 45.59 & 18,14 \\
\hline PG0832+251 & QSO & 0.329773 & $-0.22 \pm 0.005$ & 0.282 & 0.30 & 45.04 & 12,10 \\
\hline SDSSJ132704.13+443505.0 & QSO & 0.330709 & $-0.26 \pm 0.004$ & 0.0494 & 0.07 & 44.29 & 4,4 \\
\hline PG1216+069 & Sy 1 & 0.3313 & $-1.14 \pm 0.02$ & 1.10 & 1.2 & 45.63 & 20,15 \\
\hline RXJ2154.1-4414 & Sy 1 & 0.344 & $-1.32 \pm 0.001$ & 0.802 & 1.0 & 45.54 & 24,16 \\
\hline B0117-2837 & Sy 1 & 0.348858 & $-1.57 \pm 0.03$ & 1.04 & 1.2 & 45.66 & 21,17 \\
\hline PG1049-005 & Sy 1.5 & 0.3599 & $-0.72 \pm 0.02$ & 1.03 & 0.82 & 45.69 & 11,10 \\
\hline SDSSJ094952.91+390203.9 & QSO & 0.365562 & $-1.29 \pm 0.002$ & 0.677 & 0.76 & 45.53 & 11,9 \\
\hline SDSSJ132222.68+464535.2 & QSO & 0.374861 & $-1.92 \pm 0.002$ & 0.189 & 0.25 & 45.00 & 8,6 \\
\hline SDSSJ122035.10+385316.4 & Sy 1 & 0.375767 & $-0.02 \pm 0.01$ & 0.29 & 0.28 & 45.18 & 6,6 \\
\hline SDSSJ024250.85-075914.2 & QSO & 0.377651 & $-1.61 \pm 0.003$ & 0.263 & 0.33 & 45.15 & 7,6 \\
\hline SDSSJ123335.07+475800.4 & QSO & 0.38223 & $-0.59 \pm 0.009$ & 0.304 & 0.30 & 45.22 & 9,8 \\
\hline SDSSJ134246.89+184443.6 & QSO & 0.382 & $-1.57 \pm 0.004$ & 0.308 & 0.40 & 45.23 & 8,7 \\
\hline SDSSJ121037.56+315706.0 & Sy 1.2 & 0.389041 & $+0.39 \pm 0.003$ & 0.276 & 0.26 & 45.20 & 7,8 \\
\hline HB89-0202-765 & Sy 1 & 0.38939 & $-0.82 \pm 0.01$ & 0.0688 & 0.23 & 44.60 & 9,8 \\
\hline SDSSJ110312.93+414154.9 & QSO & 0.401023 & $-1.28 \pm 0.009$ & 0.186 & 0.21 & 45.06 & 9,7 \\
\hline SDSSJ133045.15+281321.4 & QSO & 0.416754 & $-1.70 \pm 0.006$ & 0.151 & 0.21 & 45.01 & 10,7 \\
\hline SDSSJ111754.31+263416.6 & QSO & 0.420466 & $-1.75 \pm 0.03$ & 0.303 & 0.38 & 45.32 & 9,7 \\
\hline SDSSJ143511.53+360437.2 & QSO & 0.428593 & $-0.58 \pm 0.3$ & 0.231 & 0.24 & 45.22 & 7,6 \\
\hline HE0435-5304 & QSO & 0.427 & $-1.31 \pm 0.0009$ & 0.179 & 0.24 & 45.12 & 12,7 \\
\hline SDSSJ110406.94+314111.4 & QSO & 0.434356 & $-1.39 \pm 0.005$ & 0.432 & 0.52 & 45.51 & 10,8 \\
\hline B0120-28 & QSO & 0.436018 & $-1.38 \pm 0.002$ & 0.558 & 0.54 & 45.62 & 15,12 \\
\hline SDSSJ161649.42+415416.3 & QSO & 0.440417 & $-0.62 \pm 0.003$ & 0.236 & 0.23 & 45.26 & 8,6 \\
\hline SDSSJ080359.23+433258.4 & Sy 1 & 0.448706 & $-0.71 \pm 0.02$ & 0.351 & 0.13 & 45.45 & 7,5 \\
\hline TON236 & Sy 1.2 & 0.4473 & $-1.02 \pm 0.003$ & 0.556 & 0.59 & 45.65 & 15,12 \\
\hline PG0003+158 & Sy 1.2 & 0.4509 & $-1.00 \pm 0.05$ & 0.769 & 0.70 & 45.80 & 20,15 \\
\hline HE0153-4520 & QSO & 0.451 & $-0.24 \pm 0.002$ & 1.43 & 1.4 & 46.07 & 22,15 \\
\hline SDSSJ100902.06+071343.8 & QSO & 0.455631 & $-1.10 \pm 0.1$ & 0.194 & 0.16 & 45.21 & 6,5 \\
\hline SDSSJ091029.75+101413.6 & QSO & 0.463194 & $-1.04 \pm 4.4$ & 0.12 & 0.07 & 45.02 & 4,5 \\
\hline SDSSJ082024.21+233450.4 & QSO & 0.470212 & $-1.25 \pm 0.01$ & 0.207 & 0.21 & 45.27 & 9,5 \\
\hline SDSSJ161916.54+334238.4 & QSO & 0.470946 & $-1.17 \pm 0.9$ & 0.523 & 0.40 & 45.67 & 12,11 \\
\hline SDSSJ092554.70+400414.1 & QSO & 0.471139 & $-0.78 \pm 0.007$ & 0.131 & 0.14 & 45.08 & 6,4 \\
\hline SDSSJ123304.05-003134.1 & QSO & 0.471167 & $-0.54 \pm 0.007$ & 0.199 & 0.22 & 45.26 & 9,6 \\
\hline PG1259+593 & Sy 1 & 0.4778 & $-0.66 \pm 0.001$ & 1.22 & 1.4 & 46.06 & 29,19 \\
\hline HE0226-4110 & Sy 1 & 0.493368 & $-1.01 \pm 0.003$ & 1.65 & 2.1 & 46.22 & 29,19 \\
\hline SDSSJ155048.29+400144.9 & QSO & 0.496843 & $-0.70 \pm 0.2$ & 0.265 & 0.19 & 45.44 & 7,6 \\
\hline HS1102+3441 & QSO & 0.508847 & $-0.82 \pm 0.008$ & 0.281 & 0.30 & 45.49 & 15,10 \\
\hline SDSSJ113327.78+032719.1 & QSO & 0.525073 & $-1.41 \pm 0.3$ & 0.117 & 0.12 & 45.14 & 7,5 \\
\hline SDSSJ025937.46+003736.3 & QSO & 0.534178 & $-0.55 \pm 0.05$ & 0.486 & 0.32 & 45.78 & 7,7 \\
\hline SDSSJ094331.61+053131.4 & QSO & 0.564336 & $-1.02 \pm 0.1$ & 0.139 & 0.17 & 45.29 & 6,4 \\
\hline SDSSJ040148.98-054056.5 & QSO & 0.570076 & $-0.39 \pm 0.03$ & 0.35 & 0.16 & 45.70 & 8,6 \\
\hline PKS0405-123 & Sy 1.2 & 0.574 & $-0.67 \pm 0.01$ & 3.69 & 3.2 & 46.73 & 63,29 \\
\hline SDSSJ124154.02+572107.3 & QSO & 0.583237 & $-1.40 \pm 0.003$ & 0.145 & 0.22 & 45.34 & 9,6 \\
\hline SDSSJ095000.73+483129.3 & Sy 1 & 0.588734 & $-1.23 \pm 0.004$ & 0.268 & 0.42 & 45.62 & 8,5 \\
\hline SDSSJ225738.20+134045.4 & QSO & 0.593937 & $-0.34 \pm 0.04$ & 0.243 & 0.19 & 45.59 & 7,6 \\
\hline SDSSJ022614.46+001529.7 & QSO & 0.6151 & $-1.51 \pm 0.01$ & 0.229 & 0.34 & 45.60 & 10,6 \\
\hline SDSSJ105945.23+144142.9 & QSO & 0.630543 & $-0.84 \pm 0.01$ & 0.327 & 0.29 & 45.78 & 10,8 \\
\hline HE0238-1904 & QSO & 0.631 & $-0.14 \pm 0.005$ & 1.17 & 1.3 & 46.34 & 23,18 \\
\hline SDSSJ111239.11+353928.2 & QSO & 0.635784 & $+0.11 \pm 0.01$ & 0.112 & 0.11 & 45.32 & 6,4 \\
\hline $3 \mathrm{C} 263$ & Sy 1.2 & 0.646 & $+0.05 \pm 0.02$ & 1.08 & 0.87 & 46.33 & 30,20 \\
\hline SDSSJ093518.19+020415.5 & QSO & 0.649117 & $-0.50 \pm 0.01$ & 0.158 & 0.17 & 45.49 & 6,5 \\
\hline PKS0637-752 & Sy 1.5 & 0.653 & $-0.16 \pm 0.1$ & 1.35 & 0.70 & 46.43 & 20,15 \\
\hline SDSSJ080908.13+461925.6 & QSO & 0.656338 & $-1.39 \pm 0.02$ & 0.637 & 0.79 & 46.11 & 12,10 \\
\hline SDSSJ105958.82+251708.8 & QSO & 0.661907 & $-1.33 \pm 0.004$ & 0.198 & 0.33 & 45.61 & 9,7 \\
\hline SDSSJ154553.48+093620.5 & QSO & 0.665 & $+0.54 \pm 1.2$ & 0.31 & -0.0 & 45.81 & 1,6 \\
\hline $3 \mathrm{C} 57$ & Sy 1.2 & 0.670527 & $-1.29 \pm 0.04$ & 0.506 & 0.77 & 46.03 & 21,12 \\
\hline PKS0552-640 & Sy 1 & 0.68 & $+0.21 \pm 0.2$ & 1.53 & 1.0 & 46.53 & 24,18 \\
\hline SDSSJ151428.64+361957.9 & QSO & 0.694596 & $-0.63 \pm 0.02$ & 0.0922 & 0.14 & 45.33 & 4,4 \\
\hline SDSSJ144511.28+342825.4 & QSO & 0.696951 & $-1.07 \pm 0.008$ & 0.0697 & 0.11 & 45.21 & 7,5 \\
\hline
\end{tabular}


Table 1

(Continued)

\begin{tabular}{|c|c|c|c|c|c|c|c|}
\hline $\begin{array}{l}\text { AGN }^{\mathrm{a}} \\
\text { Target }\end{array}$ & $\begin{array}{c}\text { AGN }^{a} \\
\text { Type }\end{array}$ & $z^{\mathrm{a}}$ & $\alpha_{\lambda}$ & $\begin{array}{c}F_{0}^{\mathrm{a}} \\
(1100 \AA)\end{array}$ & $\begin{array}{c}F_{\lambda}^{\mathrm{a}} \\
(1300 \AA)\end{array}$ & $\begin{array}{c}\log \left(\lambda L_{\lambda}\right)^{\mathrm{a}} \\
(1100 \AA)\end{array}$ & $(\mathrm{S} / \mathrm{N})_{\mathrm{res}}^{\mathrm{a}}$ \\
\hline SDSSJ113457.62+255527.9 & QSO & 0.710078 & $-0.41 \pm 0.06$ & 0.264 & 0.24 & 45.81 & 8,7 \\
\hline SDSSJ155504.39+362848.0 & QSO & 0.713654 & $-0.70 \pm 0.01$ & 0.0901 & 0.16 & 45.35 & 7,4 \\
\hline SDSSJ155304.92+354828.6 & QSO & 0.721814 & $-0.78 \pm 0.01$ & 0.409 & 0.50 & 46.02 & 8,6 \\
\hline SDSSJ091440.38+282330.6 & QSO & 0.735345 & $-1.32 \pm 0.01$ & 0.139 & 0.23 & 45.57 & 8,6 \\
\hline SDSSJ100102.55+594414.3 & QSO & 0.746236 & $-0.40 \pm 0.3$ & 0.546 & 0.58 & 46.18 & 11,9 \\
\hline SDSSJ102218.99+013218.8 & QSO & 0.789304 & $+0.01 \pm 0.03$ & 0.437 & 0.34 & 46.15 & 8,6 \\
\hline SDSSJ234500.43-005936.0 & QSO & 0.789429 & $-0.12 \pm 0.08$ & 0.188 & 0.12 & 45.78 & 7,5 \\
\hline SDSSJ101622.60+470643.3 & QSO & 0.821527 & $-1.01 \pm 0.4$ & 0.238 & 0.40 & 45.92 & 8,5 \\
\hline SBS1122+594 & QSO & 0.852 & $+0.58 \pm 0.1$ & 0.313 & 0.26 & 46.08 & 12,9 \\
\hline SDSSJ141910.20+420746.9 & QSO & 0.873501 & $-1.28 \pm 0.07$ & 0.132 & 0.21 & 45.73 & 7,4 \\
\hline SDSSJ112244.89+575543.0 & QSO & 0.905906 & $+0.11 \pm 0.005$ & 0.166 & 0.20 & 45.87 & 7,5 \\
\hline PG1148+549 & QSO & 0.975 & $-0.66 \pm 0.01$ & 0.351 & 0.46 & 46.28 & 25,15 \\
\hline SDSSJ084349.49+411741.6 & QSO & 0.989986 & $-1.10 \pm 0.2$ & 0.104 & 0.08 & 45.77 & 5,5 \\
\hline HE0439-5254 & QSO & 1.053 & $-1.05 \pm 0.04$ & 0.241 & 0.38 & 46.20 & 14,9 \\
\hline SDSS-J100535.24+013445.7 & QSO & 1.0809 & $-1.87 \pm 0.09$ & 0.143 & 0.27 & 46.00 & 11,11 \\
\hline FIRST-J020930.7-043826 & QSO & 1.131 & $-2.06 \pm 0.02$ & 0.0858 & 0.25 & 45.82 & 13,13 \\
\hline PG1206+459 & QSO & 1.16254 & $-0.81 \pm 1.1$ & 0.296 & 0.40 & 46.39 & 21,16 \\
\hline PG1338+416 & QSO & 1.21422 & $-1.94 \pm 0.4$ & 0.0632 & 0.18 & 45.77 & 16,11 \\
\hline LBQS-1435-0134 & QSO & 1.30791 & $-0.65 \pm 0.01$ & 0.418 & 0.59 & 46.67 & 26,20 \\
\hline PG1522+101 & QSO & 1.32785 & $-0.79 \pm 0.3$ & 0.372 & 0.43 & 46.64 & 19,14 \\
\hline Q0232-042 & QSO & 1.43737 & $-0.83 \pm 0.3$ & 0.158 & 0.22 & 46.35 & 15,12 \\
\hline PG1630+377 & QSO & 1.47607 & $-1.95 \pm 0.1$ & 0.176 & 0.70 & 46.43 & 25,10 \\
\hline
\end{tabular}

Notes. ${ }^{a}$ Our 159 AGN targets, types, redshifts, fluxes, spectral indices, luminosities, and S/N ratios. All fluxes in units of $10^{-14} \mathrm{erg} \mathrm{cm}^{-2} \mathrm{~s}^{-1} \AA^{-1}$. Rest-frame, dereddened spectral distributions are fitted to power laws, $F_{\lambda}=F_{0}(\lambda / 1100 \AA)^{\alpha_{\lambda}}$. Wavelength index $\alpha_{\lambda}$ corresponds to frequency index $\alpha_{\nu}=-\left[2+\alpha_{\lambda}\right]$. The eight columns show 1: AGN target; 2: AGN type; 3: AGN redshift; 4: fitted spectral index, $\alpha_{\lambda}$, with statistical errors; 5: rest-frame flux normalization $F_{0}$ at $1100 \AA$; 6: observed flux $F_{\lambda}$ at $1300 \AA$; 7: band luminosity, $\lambda L_{\lambda}$ at $1100 \AA$ (in $\mathrm{erg} \mathrm{s}^{-1}$ ); and 8: signal-to-noise at $1250 \AA$ and $1550 \AA$ for data with G130M (1132-1460 ) and G160M (1394-1798 $\AA$ ) gratings, respectively. Flux at $1300 \AA$ for SBS $1108+560$ (noted with *) is low, owing to LyC absorption ( $\lambda<1334 \AA$ ) from a LLS at $z=0.46335$. Finn et al. (2014) fitted a harder spectral index, $\alpha_{\lambda}=-0.64$, for J0209-0438 at $z=1.131$, using additional COS/G230L spectra extending to longer wavelengths.

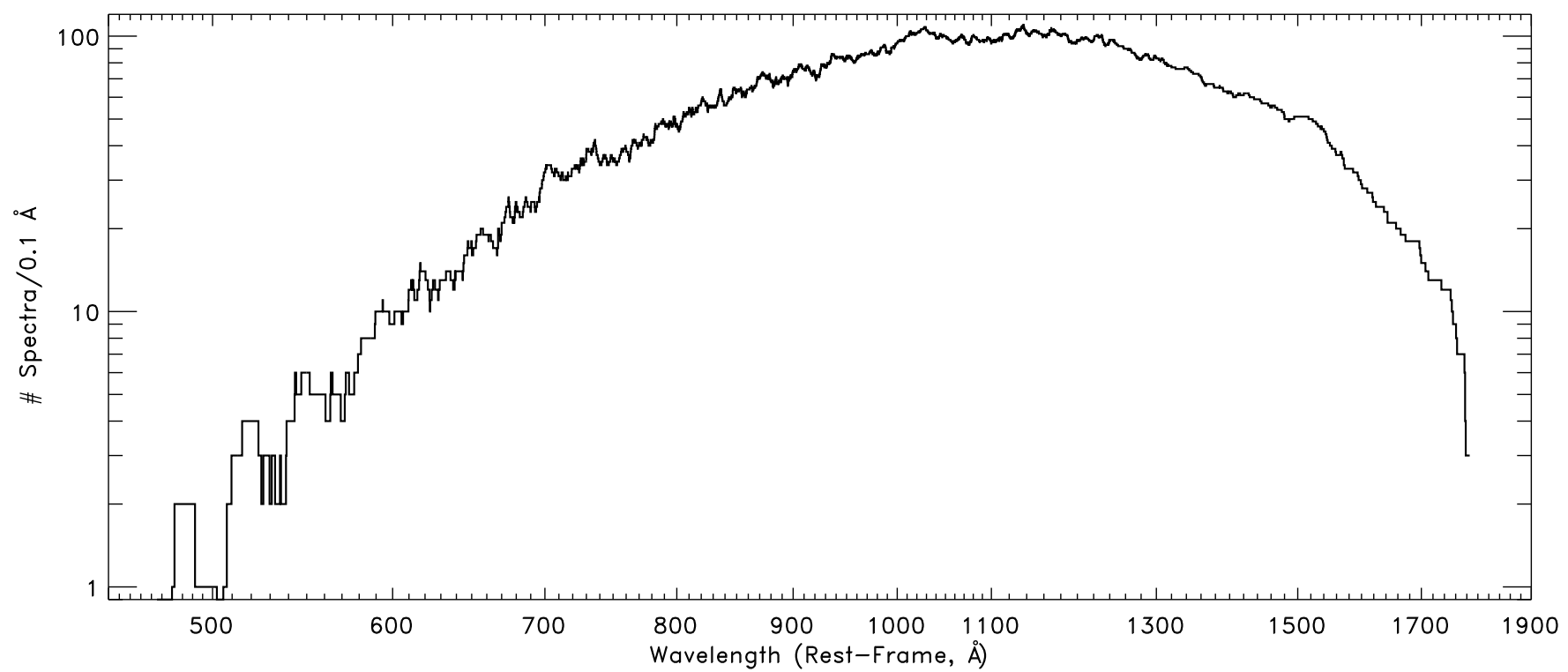

Figure 2. Number of AGN targets that contribute to the composite spectrum (see Figure 5) as a function of AGN rest-frame wavelength. Note the rapid decline in targets that probe short wavelengths, with 10 or fewer AGNs probing $\lambda \leqslant 600 \AA$. 
Telescopes (MAST) and then aligned and coadded using IDL procedures developed by the COS GTO team. ${ }^{4}$ Typical wavelength shifts were a resolution element $(\sim 0.1 \AA)$ or less, and the coadded flux in each pixel was calculated as the exposure-weighed mean of the flux in aligned exposures.

2. Fit spline functions to spectra. The raw data contain narrow absorption features that should be excluded from the AGN composite spectrum. Identifying and masking each of these features by hand in all of our spectra would be extremely tedious. For this reason, we utilize a semi-automated routine that removes narrow absorption features and fits the remaining data with a piecewisecontinuous function composed of spline functions and Legendre polynomial functions. This spline-fitting process involves first splitting the spectra into 5-10 $\AA$ segments and calculating the average $\mathrm{S} / \mathrm{N}$ per pixel (flux/error) in each segment. Pixels with $\mathrm{S} / \mathrm{N}$ less than $1.5 \sigma$ below their segment $\mathrm{S} / \mathrm{N}$ are rejected from the fitting process to exclude absorption features and regions of increased noise. This process is repeated iteratively until there is little change between iterations. The median flux values in the segments are then fitted with a spline function. We manually inspect the fits and adjust the identification of rejected regions as necessary. Smoothly varying data are well described by this spline-only method. Near broad emission and other cusp-like features, short segments of piecewise-continuous Legendre polynomials are preferred. More details on the process are given in our IGM survey paper (Danforth et al. 2014).

3. Deredden spectra. We correct the fine-grained data and their corresponding spline fits for Galactic reddening, using the empirical mean extinction curve of Fitzpatrick (1999) with a ratio of total-to-selective extinction $R_{V}=A_{V} / E(B-V)=$ 3.1 and color excesses $E(B-V)$ from NED. In this paper, we use values of $E(B-V)$ based on dust mapping by Schlegel et al. (1998) with a $14 \%$ recalibration by Schlafly \& Finkbeiner (2011). We do not correct for reddening intrinsic to the AGNs, although we do not think this could be a substantial effect. We can probably rule out a large amount of dust (see discussion in Section 3.2).

4. Identify pLLS and LLS absorption. In Paper I, we identified pLLS absorption by inspecting the spectra for flux decrements or Lyman breaks. For this paper, we employ a custom computer script that scans each spectrum for correlated down-pixels at the locations of higher-order Lyman lines of pLLS and LLS absorbers. First, the script divides the spectra by their respective spline fits, normalizing the flux unaffected by IGM absorption to unity. We determine the median flux for 15 pixels that have the same relative spacing as the first $15 \mathrm{HI}$ Lyman lines of a pLLS with a redshift equal to the source AGN. If there is a pLLS, the median will be much less than unity. We then step one pixel to the left, recalculate the relative spacing of the first 15 Lyman lines at this redshift and the median flux for this group of 15 pixels. We repeat this process until we reach the end of the spectrum or a pLLS redshift of zero. The script returns a list of redshifts of system candidates to be inspected. When a system is confirmed, we measure the equivalent widths of up to the first 12 Lyman lines and fit them to a curve of growth $(\mathrm{CoG})$ to determine the column

\footnotetext{
4 IDL routines available at

http://casa.colorado.edu/ danforth/science/cos/costools.
}

density and Doppler parameter of the system. In Paper I, we found a total of 17 LLS and pLLS systems in 8 of the 22 sight lines, and we were sensitive to systems with column density $\log N_{\mathrm{H}_{\mathrm{I}}} \geqslant 15.5$. In this paper, using our new identification method, we confirm the 17 previously identified systems plus 13 unidentified systems above the sensitivity limit $\log N_{\mathrm{H}_{\mathrm{I}}} \sim 15.5$ in the same 22 sight lines from Paper I. Figure 3 shows examples of pLLS identification and continuum restoration. The lowest column density measurement derived from CoG fitting in this paper is $\log N_{\mathrm{HI}} \sim 13.4$. We detect 221 systems (7 LLS and 214 pLLS) in 71 of the 159 AGN sight lines, with absorber redshifts $0.24332 \leqslant z_{a} \leqslant 0.91449$. These absorbers are listed in Table 2 together with our measurements of their redshifts, H I column densities, and Doppler parameters. Of the 221 systems, 167 have column densities $\log N_{\mathrm{H}_{\mathrm{I}}} \geqslant 15.0$ whose distribution in column density is shown in Figure 4. We only correct for these 167 systems in our analysis. Systems with $\log N_{\mathrm{H}_{\mathrm{I}}}=15.5$ and $\log N_{\mathrm{H}}=15.0$ have opacity that depress the flux immediately blueward of the Lyman limit by less than $2 \%$ and $0.7 \%$ respectively. Owing to the multiple correlated Lyman lines used in this identification technique, our sensitivity is better than the local $\mathrm{S} / \mathrm{N}$ over most of the spectral coverage. Correcting for the opacity of weaker systems $\left(\log N_{\mathrm{H}_{\mathrm{I}}}<15.0\right)$ would have a negligible effect on our analysis of AGN continuum, changing the EUV slope of our composite spectrum by only 0.006 .

5. Restore flux depressed by pLLS and mask unrecoverable flux. We account for Lyman continuum absorption by measuring the equivalent widths of the first 12 Lyman lines and fitting them with a $\mathrm{CoG}$ to estimate the $\mathrm{HI}_{\mathrm{I}}$ column density and Doppler parameter. We use these measurements to correct for the $v^{-3}$ opacity shortward of each Lyman edge. We correct only the flux below the Lyman limit. When a spectrum has pLLS absorption with column density $\log N_{\mathrm{HI}}=15.0-15.9$, we mask the flux between the Lyman limit (911.753 $\AA$ ) and Lyman-9 (916.429 $\AA$ ) or $\sim 4.7 \AA$ redward in the pLLS rest frame. For a pLLS with $\log N_{\mathrm{HI}} \geqslant 15.9$, we mask from the Lyman limit to Lyman$13(920.963 \AA)$ or $\sim 9.2 \AA$ redward. When data blueward of the Lyman limit of LLS or pLLS had S/N $<1$ or did not appear continuous with two or more regions of continuum redward of the Lyman limit, we masked the data. We also mask regions of the spectra affected by broad absorption from damped Ly $\alpha$ systems and $\mathrm{H}_{2}$ Lyman bands after qualitative visual inspection. The amount of masking varies for individual cases. Some spectra have one or two gaps of $\leqslant 10 \AA$ in the data from observations that were not planned with contiguous wavelength coverage over the entire COS-FUV spectral range. These gaps are masked prior to our continuum analysis.

6. Shift to rest-frame. We shift each spectrum to the rest-frame of the AGNs by dividing the wavelength array by $\left(1+z_{\mathrm{AGN}}\right)$.

7. Mask non-AGN features. In every spectrum, we exclude Galactic Ly $\alpha$ absorption (1215.67 $\AA$ ) by masking $14 \AA$ on both sides of the line center in the observed frame. We exclude geocoronal emission lines of $\mathrm{N}_{\mathrm{I}} \lambda 1200$ and $\mathrm{O}_{\mathrm{I}}$ $\lambda 1304$ by masking $2 \AA$ on both sides of $\mathrm{N}_{\mathrm{I}}$ and $5 \AA$ on both sides of $\mathrm{O}$ I. In five spectra, we masked the absorption due to the $\operatorname{Ly} \alpha$ line of damped $\operatorname{Ly} \alpha$ systems.

8. Resample the spectra. As in Paper I and Telfer et al. (2002), we resample the spectra to uniform $0.1 \AA$ bins. After resampling, each flux pixel corresponds to a new 

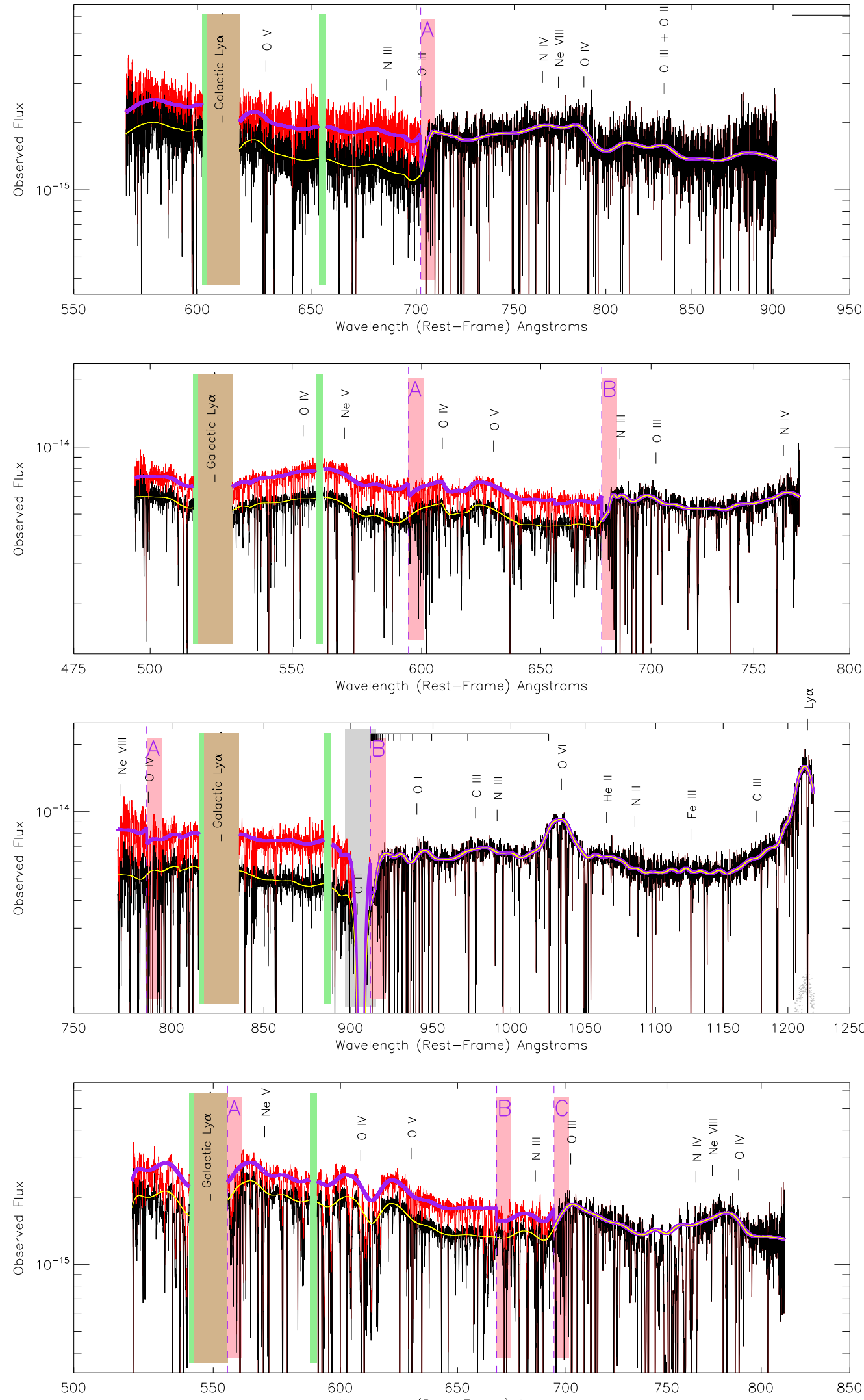





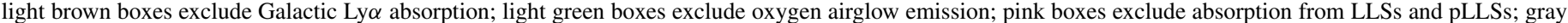

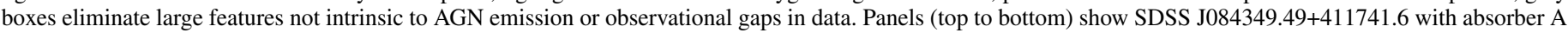

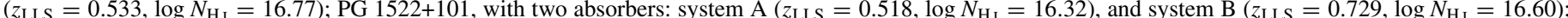


system A $\left(z_{\mathrm{LLS}}=0.349, \log N_{\mathrm{HI}}=16.37\right)$, system B $\left(z_{\mathrm{LLS}}=0.621, \log N_{\mathrm{H}_{\mathrm{I}}}=16.30\right)$, and system C $\left(z_{\mathrm{LLS}}=0.686, \log N_{\mathrm{HI}}=16.49\right)$.

(A color version of this figure is available in the online journal.) 
Table 2

Lyman-limit Systems and Partial Lyman-limit Systems

\begin{tabular}{|c|c|c|c|}
\hline$\overline{\text { AGN Target }}$ & $\begin{array}{c}z_{\text {LLS }}^{\mathrm{a}} \\
\text { (redshift) }\end{array}$ & $\begin{array}{c}\log N_{\mathrm{HI}_{\mathrm{I}}}^{\mathrm{a}} \\
\left(N_{\mathrm{H}_{\mathrm{I}}} \text { in } \mathrm{cm}^{-2}\right)\end{array}$ & $\begin{array}{c}b^{\mathrm{a}} \\
\left(\mathrm{km} \mathrm{s}^{-1}\right)\end{array}$ \\
\hline SDSSJ115758.72-002220.8 & 0.25661 & $15.25 \pm 0.03$ & 25 \\
\hline \multirow[t]{2}{*}{ SDSSJ092554.43+453544.4 } & 0.25057 & $15.14 \pm 0.03$ & 25 \\
\hline & 0.30959 & $15.27 \pm 0.03$ & 25 \\
\hline PG1216+069 & 0.28231 & $16.29 \pm 0.01$ & 34 \\
\hline \multirow[t]{2}{*}{ B0117-2837 } & 0.34833 & $15.52 \pm 0.05$ & 25 \\
\hline & 0.34866 & $16.02 \pm 0.02$ & 25 \\
\hline SDSSJ122035.10+385316.4 & 0.27332 & $15.61 \pm 0.02$ & 25 \\
\hline SDSSJ123335.07+475800.4 & 0.28495 & $15.41 \pm 0.03$ & 25 \\
\hline HB89-0202-765 & 0.30657 & $14.95 \pm 0.03$ & 25 \\
\hline SDSSJ110312.93+414154.9 & 0.27116 & $15.11 \pm 0.03$ & 25 \\
\hline SDSSJ133045.15+281321.4 & 0.27553 & $15.20 \pm 0.02$ & 25 \\
\hline SDSSJ111754.31+263416.6 & 0.35193 & $16.14 \pm 0.02$ & 25 \\
\hline \multirow[t]{3}{*}{ SDSSJ143511.53+360437.2 } & 0.26246 & $15.24 \pm 0.02$ & 25 \\
\hline & 0.37292 & $16.72 \pm 0.06$ & 25 \\
\hline & 0.3876 & $16.15 \pm 0.02$ & 25 \\
\hline \multirow[t]{8}{*}{ PG0003+158 } & 0.30573 & $15.47 \pm 0.01$ & 25 \\
\hline & 0.31215 & $14.38 \pm 0.02$ & 25 \\
\hline & 0.34787 & $15.98 \pm 0.04$ & 17 \\
\hline & 0.36619 & $15.10 \pm 0.02$ & 25 \\
\hline & 0.37034 & $14.68 \pm 0.06$ & 25 \\
\hline & 0.38612 & $14.84 \pm 0.01$ & 25 \\
\hline & 0.40137 & $15.04 \pm 0.02$ & 25 \\
\hline & 0.42184 & $14.77 \pm 0.02$ & 25 \\
\hline HE0153-4520 & 0.40051 & $14.52 \pm 0.02$ & 25 \\
\hline \multirow[t]{5}{*}{ SDSSJ100902.06+071343.8 } & 0.35586 & $17.41 \pm 0.04$ & 25 \\
\hline & 0.3745 & $14.28 \pm 0.06$ & 25 \\
\hline & 0.37554 & $15.62 \pm 0.04$ & 25 \\
\hline & 0.37624 & $14.86 \pm 0.06$ & 25 \\
\hline & 0.41401 & $15.15 \pm 0.02$ & 25 \\
\hline \multirow[t]{4}{*}{ SDSSJ091029.75+101413.6 } & 0.2634 & $16.86 \pm 0.5$ & 25 \\
\hline & 0.26375 & $14.17 \pm 0.1$ & 25 \\
\hline & 0.26432 & $14.96 \pm 0.03$ & 25 \\
\hline & 0.41924 & $17.14 \pm 0.8$ & 29 \\
\hline SDSSJ082024.21+233450.4 & 0.45424 & $14.56 \pm 0.02$ & 25 \\
\hline \multirow[t]{8}{*}{ SDSSJ161916.54+334238.4 } & 0.2694 & $16.40 \pm 0.03$ & 25 \\
\hline & 0.26988 & $14.72 \pm 0.08$ & 25 \\
\hline & 0.27086 & $14.85 \pm 0.02$ & 25 \\
\hline & 0.2716 & $14.58 \pm 0.08$ & 25 \\
\hline & 0.42676 & $14.93 \pm 0.07$ & 25 \\
\hline & 0.44231 & $15.81 \pm 0.02$ & 25 \\
\hline & 0.47091 & $16.84 \pm 0.1$ & 25 \\
\hline & 0.47179 & $14.47 \pm 0.03$ & 25 \\
\hline \multirow[t]{2}{*}{ SDSSJ092554.70+400414.1 } & 0.2477 & $19.26 \pm 0.06$ & 59 \\
\hline & 0.25283 & $14.84 \pm 0.05$ & 25 \\
\hline \multirow[t]{2}{*}{ SDSSJ123304.05-003134.1 } & 0.31875 & $15.51 \pm 0.01$ & 25 \\
\hline & 0.43061 & $14.89 \pm 0.02$ & 25 \\
\hline PG1259+593 & 0.2924 & $14.49 \pm 0.02$ & 25 \\
\hline \multirow[t]{2}{*}{ HE0226-4110 } & 0.24525 & $14.24 \pm 0.03$ & 25 \\
\hline & 0.49252 & $14.64 \pm 0.05$ & 25 \\
\hline \multirow[t]{4}{*}{ SDSSJ155048.29+400144.9 } & 0.31257 & $16.62 \pm 0.06$ & 41 \\
\hline & 0.42739 & $15.64 \pm 0.02$ & 25 \\
\hline & 0.4919 & $16.57 \pm 0.02$ & 25 \\
\hline & 0.49255 & $15.69 \pm 0.05$ & 25 \\
\hline \multirow[t]{6}{*}{ HS1102+3441 } & 0.26164 & $14.95 \pm 0.03$ & 25 \\
\hline & 0.28916 & $14.49 \pm 0.04$ & 25 \\
\hline & 0.28986 & $14.89 \pm 0.03$ & 25 \\
\hline & 0.31039 & $15.07 \pm 0.01$ & 25 \\
\hline & 0.33246 & $14.37 \pm 0.02$ & 25 \\
\hline & 0.50607 & $15.07 \pm 0.02$ & 25 \\
\hline \multirow{3}{*}{ SDSSJ113327.78+032719.1 } & 0.24663 & $17.53 \pm 0.1$ & 25 \\
\hline & 0.30216 & $14.44 \pm 0.04$ & 25 \\
\hline & 0.45225 & $15.22 \pm 0.03$ & 25 \\
\hline SDSSJ094331.61+053131.4 & 0.35464 & $16.12 \pm 0.09$ & 91 \\
\hline \multirow[t]{2}{*}{ SDSSJ040148.98-054056.5 } & 0.32381 & $15.37 \pm 0.01$ & 25 \\
\hline & 0.36547 & $14.60 \pm 0.05$ & 25 \\
\hline
\end{tabular}

Table 2

(Continued)

\begin{tabular}{|c|c|c|c|}
\hline AGN Target & $\begin{array}{c}z_{\mathrm{LLS}}^{\mathrm{a}} \\
\text { (redshift) }\end{array}$ & $\begin{array}{c}\log N_{\mathrm{H}_{\mathrm{I}}}^{\mathrm{a}} \\
\left(N_{\mathrm{H}_{\mathrm{I}}} \text { in } \mathrm{cm}^{-2}\right)\end{array}$ & $\begin{array}{c}b^{\mathrm{a}} \\
\left(\mathrm{km} \mathrm{s}^{-1}\right)\end{array}$ \\
\hline \multirow[t]{2}{*}{ PKS0405-123 } & 0.36077 & $15.04 \pm 0.02$ & 25 \\
\hline & 0.4057 & $14.83 \pm 0.02$ & 25 \\
\hline SDSSJ095000.73+483129.3 & 0.48502 & $15.07 \pm 0.03$ & 25 \\
\hline \multirow[t]{3}{*}{ SDSSJ225738.20+134045.4 } & 0.37712 & $15.07 \pm 0.02$ & 25 \\
\hline & 0.3787 & $14.90 \pm 0.08$ & 25 \\
\hline & 0.49905 & $15.72 \pm 0.02$ & 25 \\
\hline SDSSJ022614.46+001529.7 & 0.4161 & $14.77 \pm 0.02$ & 25 \\
\hline \multirow[t]{4}{*}{ SDSSJ105945.23+144142.9 } & 0.34074 & $15.33 \pm 0.02$ & 25 \\
\hline & 0.46567 & $15.65 \pm 0.03$ & 25 \\
\hline & 0.57638 & $15.51 \pm 0.03$ & 25 \\
\hline & 0.61727 & $14.71 \pm 0.02$ & 25 \\
\hline \multirow[t]{4}{*}{ HE0238-1904 } & 0.3441 & $14.69 \pm 0.02$ & 25 \\
\hline & 0.35534 & $14.87 \pm 0.03$ & 25 \\
\hline & 0.40102 & $14.91 \pm 0.02$ & 25 \\
\hline & 0.42424 & $15.03 \pm 0.02$ & 25 \\
\hline SDSSJ111239.11+353928.2 & 0.24679 & $15.45 \pm 0.03$ & 25 \\
\hline \multirow[t]{2}{*}{$3 \mathrm{C} 263$} & 0.32545 & $15.44 \pm 0.02$ & 25 \\
\hline & 0.52796 & $15.55 \pm 0.01$ & 25 \\
\hline \multirow[t]{2}{*}{ SDSSJ093518.19+020415.5 } & 0.35457 & $15.26 \pm 0.03$ & 25 \\
\hline & 0.42852 & $14.69 \pm 0.03$ & 25 \\
\hline \multirow[t]{4}{*}{ PKS0637-752 } & 0.24326 & $15.86 \pm 0.09$ & 38 \\
\hline & 0.41755 & $15.42 \pm 0.01$ & 25 \\
\hline & 0.4528 & $15.47 \pm 0.02$ & 25 \\
\hline & 0.46847 & $16.08 \pm 0.03$ & 25 \\
\hline SDSSJ080908.13+461925.6 & 0.61917 & $16.15 \pm 0.01$ & 25 \\
\hline \multirow[t]{2}{*}{ SDSSJ154553.48+093620.5 } & 0.47379 & $17.25 \pm 0.2$ & 25 \\
\hline & 0.47623 & $15.62 \pm 0.02$ & 25 \\
\hline \multirow[t]{6}{*}{$3 \mathrm{C} 57$} & 0.24988 & $15.67 \pm 0.009$ & 25 \\
\hline & 0.29224 & $14.84 \pm 0.02$ & 25 \\
\hline & 0.32332 & $16.29 \pm 0.01$ & 76 \\
\hline & 0.32827 & $15.53 \pm 0.02$ & 25 \\
\hline & 0.38329 & $14.75 \pm 0.02$ & 25 \\
\hline & 0.5332 & $14.22 \pm 0.08$ & 25 \\
\hline \multirow[t]{4}{*}{ PKS0552-640 } & 0.34517 & $16.71 \pm 0.03$ & 25 \\
\hline & 0.34592 & $14.18 \pm 0.04$ & 25 \\
\hline & 0.446 & $15.89 \pm 0.04$ & 30 \\
\hline & 0.63017 & $14.78 \pm 0.06$ & 25 \\
\hline SDSSJ151428.64+361957.9 & 0.41065 & $17.93 \pm 0.2$ & 42 \\
\hline SDSSJ144511.28+342825.4 & 0.60722 & $15.51 \pm 0.02$ & 25 \\
\hline \multirow[t]{4}{*}{ SDSSJ113457.62+255527.9 } & 0.2469 & $14.66 \pm 0.03$ & 25 \\
\hline & 0.43233 & $16.40 \pm 0.03$ & 53 \\
\hline & 0.50265 & $15.19 \pm 0.02$ & 25 \\
\hline & 0.66824 & $15.01 \pm 0.05$ & 25 \\
\hline \multirow[t]{4}{*}{ SDSSJ155504.39+362848.0 } & 0.30689 & $14.57 \pm 0.02$ & 25 \\
\hline & 0.36504 & $14.94 \pm 0.02$ & 25 \\
\hline & 0.57611 & $15.52 \pm 0.03$ & 25 \\
\hline & 0.60275 & $15.36 \pm 0.02$ & 25 \\
\hline \multirow[t]{9}{*}{ SDSSJ124511.25+335610.1 } & 0.31802 & $14.80 \pm 0.03$ & 25 \\
\hline & 0.44947 & $15.44 \pm 0.1$ & 25 \\
\hline & 0.55682 & $16.50 \pm 0.2$ & 25 \\
\hline & 0.58762 & $15.14 \pm 0.03$ & 25 \\
\hline & 0.63215 & $15.92 \pm 0.06$ & 65 \\
\hline & 0.64496 & $15.70 \pm 0.04$ & 25 \\
\hline & 0.64862 & $15.17 \pm 0.04$ & 25 \\
\hline & 0.68918 & $16.68 \pm 0.2$ & 25 \\
\hline & 0.71297 & $16.32 \pm 0.1$ & 35 \\
\hline \multirow[t]{2}{*}{ SDSSJ155304.92+354828.6 } & 0.4756 & $15.43 \pm 0.03$ & 25 \\
\hline & 0.52027 & $15.18 \pm 0.03$ & 25 \\
\hline SDSSJ091440.38+282330.6 & 0.24426 & $15.39 \pm 0.01$ & 25 \\
\hline & 0.59969 & $15.33 \pm 0.02$ & 25 \\
\hline SDSSJ100102.55+594414.3 & 0.30355 & $17.27 \pm 0.04$ & 25 \\
\hline & 0.4159 & $16.61 \pm 0.02$ & 25 \\
\hline SBS1108+560 & 0.28646 & $15.91 \pm 0.2$ & 50 \\
\hline & 0.46334 & $17.06 \pm 0.1$ & 35 \\
\hline & 0.61765 & $15.07 \pm 0.03$ & 25 \\
\hline
\end{tabular}


Table 2

(Continued)

\begin{tabular}{|c|c|c|c|}
\hline AGN Target & $\begin{array}{c}z_{\mathrm{LLS}}^{\mathrm{a}} \\
\text { (redshift) }\end{array}$ & $\begin{array}{c}\log N_{\mathrm{HI}_{\mathrm{I}}}^{\mathrm{a}} \\
\left(N_{\mathrm{H}_{\mathrm{I}}} \text { in } \mathrm{cm}^{-2}\right)\end{array}$ & $\begin{array}{c}b^{\mathrm{a}} \\
\left(\mathrm{km} \mathrm{s}^{-1}\right)\end{array}$ \\
\hline & 0.68267 & $15.36 \pm 0.02$ & 25 \\
\hline \multirow{5}{*}{ SDSSJ143726.14+504555.8 } & 0.25065 & $15.77 \pm 0.06$ & 25 \\
\hline & 0.56945 & $15.23 \pm 0.03$ & 25 \\
\hline & 0.76901 & $15.52 \pm 0.08$ & 25 \\
\hline & 0.77109 & $14.94 \pm 0.1$ & 25 \\
\hline & 0.77248 & $16.07 \pm 0.1$ & 36 \\
\hline \multirow{2}{*}{ SDSSJ102218.99+013218.8 } & 0.39907 & $13.41 \pm 0.09$ & 25 \\
\hline & 0.7425 & $15.35 \pm 0.1$ & 25 \\
\hline \multirow{2}{*}{ SDSSJ234500.43-005936.0 } & 0.2539 & $16.08 \pm 0.03$ & 25 \\
\hline & 0.54818 & $15.96 \pm 0.07$ & 34 \\
\hline \multirow[t]{4}{*}{ SDSSJ101622.60+470643.3 } & 0.4321 & $15.59 \pm 0.02$ & 25 \\
\hline & 0.66475 & $15.92 \pm 0.1$ & 25 \\
\hline & 0.72766 & $16.26 \pm 0.3$ & 14 \\
\hline & 0.74627 & $15.43 \pm 0.05$ & 25 \\
\hline \multirow[t]{8}{*}{ SBS1122+594 } & 0.31236 & $14.95 \pm 0.02$ & 25 \\
\hline & 0.31784 & $14.43 \pm 0.04$ & 25 \\
\hline & 0.35115 & $15.14 \pm 0.02$ & 25 \\
\hline & 0.3919 & $15.18 \pm 0.06$ & 25 \\
\hline & 0.55744 & $15.82 \pm 0.03$ & 25 \\
\hline & 0.55817 & $16.42 \pm 0.02$ & 25 \\
\hline & 0.5698 & $14.72 \pm 0.04$ & 25 \\
\hline & 0.67835 & $15.97 \pm 0.08$ & 19 \\
\hline \multirow{7}{*}{ SDSSJ141910.20+420746.9 } & 0.289 & $16.17 \pm 0.03$ & 25 \\
\hline & 0.42561 & $16.02 \pm 0.02$ & 25 \\
\hline & 0.52221 & $15.87 \pm 0.02$ & 25 \\
\hline & 0.53461 & $16.06 \pm 0.07$ & 25 \\
\hline & 0.60842 & $15.72 \pm 0.02$ & 25 \\
\hline & 0.80463 & $15.95 \pm 0.04$ & 25 \\
\hline & 0.84523 & $16.20 \pm 0.03$ & 25 \\
\hline SDSSJ112244.89+575543.0 & 0.39798 & $14.78 \pm 0.03$ & 25 \\
\hline \multirow[t]{3}{*}{ FBQSJ0751+2919 } & 0.43187 & $15.69 \pm 0.01$ & 25 \\
\hline & 0.49455 & $15.56 \pm 0.02$ & 25 \\
\hline & 0.82902 & $15.99 \pm 0.02$ & 25 \\
\hline \multirow[t]{6}{*}{ PG1407+265 } & 0.29717 & $15.13 \pm 0.03$ & 25 \\
\hline & 0.32569 & $15.20 \pm 0.01$ & 25 \\
\hline & 0.57488 & $15.56 \pm 0.01$ & 25 \\
\hline & 0.59964 & $15.78 \pm 0.03$ & 26 \\
\hline & 0.68278 & $16.39 \pm 0.03$ & 33 \\
\hline & 0.81699 & $15.62 \pm 0.02$ & 25 \\
\hline \multirow[t]{5}{*}{ HB89-0107-025-NED05 } & 0.39909 & $16.59 \pm 0.02$ & 30 \\
\hline & 0.53546 & $15.08 \pm 0.03$ & 25 \\
\hline & 0.7178 & $15.37 \pm 0.01$ & 25 \\
\hline & 0.8093 & $15.17 \pm 0.05$ & 25 \\
\hline & 0.87569 & $15.51 \pm 0.06$ & 25 \\
\hline \multirow[t]{3}{*}{ LBQS-0107-0235 } & 0.53635 & $15.81 \pm 0.05$ & 86 \\
\hline & 0.71892 & $15.54 \pm 0.02$ & 25 \\
\hline & 0.87636 & $15.77 \pm 0.02$ & 25 \\
\hline \multirow[t]{4}{*}{ PG1148+549 } & 0.25242 & $15.26 \pm 0.02$ & 25 \\
\hline & 0.57785 & $15.24 \pm 0.01$ & 25 \\
\hline & 0.68864 & $15.52 \pm 0.03$ & 25 \\
\hline & 0.90485 & $15.34 \pm 0.05$ & 25 \\
\hline \multirow[t]{4}{*}{ SDSSJ084349.49+411741.6 } & 0.53258 & $16.67 \pm 0.05$ & 25 \\
\hline & 0.53346 & $16.10 \pm 0.03$ & 25 \\
\hline & 0.54106 & $15.30 \pm 0.02$ & 25 \\
\hline & 0.54353 & $15.57 \pm 0.04$ & 25 \\
\hline \multirow{3}{*}{ HE0439-5254 } & 0.328 & $15.67 \pm 0.01$ & 25 \\
\hline & 0.61508 & $16.25 \pm 0.04$ & 52 \\
\hline & 0.86515 & $15.58 \pm 0.03$ & 25 \\
\hline \multirow[t]{5}{*}{ SDSS-J100535.24+013445.7 } & 0.41753 & $15.04 \pm 0.05$ & 25 \\
\hline & 0.41853 & $16.37 \pm 0.03$ & 25 \\
\hline & 0.41963 & $15.83 \pm 0.02$ & 25 \\
\hline & 0.83711 & $16.81 \pm 0.01$ & 25 \\
\hline & 0.83938 & $16.10 \pm 0.04$ & 25 \\
\hline FIRST-J020930.7-043826 & 0.39035 & $18.00 \pm 0.2$ & 49 \\
\hline & 0.8268 & $15.05 \pm 0.04$ & 25 \\
\hline
\end{tabular}

Table 2

(Continued)

\begin{tabular}{|c|c|c|c|}
\hline AGN Target & $\begin{array}{c}z_{\mathrm{LLS}}^{\mathrm{a}} \\
\text { (redshift) }\end{array}$ & $\begin{array}{c}\log N_{\mathrm{H}_{\mathrm{I}}}^{\mathrm{a}} \\
\left(N_{\mathrm{H}_{\mathrm{I}}} \text { in } \mathrm{cm}^{-2}\right)\end{array}$ & $\begin{array}{c}b^{\mathrm{a}} \\
\left(\mathrm{km} \mathrm{s}^{-1}\right)\end{array}$ \\
\hline \multirow[t]{3}{*}{ PG1206+459 } & 0.40852 & $15.71 \pm 0.02$ & 25 \\
\hline & 0.41412 & $15.69 \pm 0.04$ & 25 \\
\hline & 0.92772 & $17.03 \pm 0.08$ & 46 \\
\hline \multirow[t]{4}{*}{ PG1338+416 } & 0.34886 & $16.37 \pm 0.06$ & 41 \\
\hline & 0.46369 & $15.28 \pm 0.01$ & 25 \\
\hline & 0.62136 & $16.30 \pm 0.05$ & 58 \\
\hline & 0.68617 & $16.49 \pm 0.04$ & 32 \\
\hline \multirow[t]{6}{*}{ LBQS-1435-0134 } & 0.26228 & $14.96 \pm 0.02$ & 25 \\
\hline & 0.29907 & $15.30 \pm 0.02$ & 25 \\
\hline & 0.39214 & $15.03 \pm 0.02$ & 25 \\
\hline & 0.43834 & $14.76 \pm 0.02$ & 25 \\
\hline & 0.61283 & $15.30 \pm 0.02$ & 25 \\
\hline & 0.68124 & $15.54 \pm 0.02$ & 25 \\
\hline \multirow[t]{4}{*}{ PG-1522+101 } & 0.51841 & $16.32 \pm 0.2$ & 16 \\
\hline & 0.57179 & $15.31 \pm 0.06$ & 17 \\
\hline & 0.67518 & $15.87 \pm 0.01$ & 25 \\
\hline & 0.72879 & $16.60 \pm 0.09$ & 26 \\
\hline \multirow[t]{3}{*}{ Q0232-042 } & 0.32239 & $16.14 \pm 0.03$ & 30 \\
\hline & 0.73888 & $16.64 \pm 0.08$ & 35 \\
\hline & 0.80773 & $15.60 \pm 0.05$ & 32 \\
\hline \multirow[t]{6}{*}{ PG1630+377 } & 0.27395 & $16.92 \pm 0.04$ & 44 \\
\hline & 0.27821 & $14.72 \pm 0.02$ & 25 \\
\hline & 0.41774 & $15.72 \pm 0.02$ & 25 \\
\hline & 0.41856 & $14.67 \pm 0.04$ & 25 \\
\hline & 0.8111 & $15.52 \pm 0.03$ & 25 \\
\hline & 0.91449 & $15.81 \pm 0.01$ & 25 \\
\hline
\end{tabular}

Notes. ${ }^{\text {a }}$ In these $71 \mathrm{AGN}$ sight lines, we find 7 Lyman-limit systems $\left(\log N_{\mathrm{H}_{\mathrm{I}}} \geqslant\right.$ 17.2) and 214 partial Lyman-limit absorbers $\left(15.0 \leqslant \log N_{\mathrm{H}_{\mathrm{I}}}<17.2\right)$. We list their redshifts, H I column densities, lower limit statistical uncertainties, and Doppler parameters $(b)$ derived by fitting $\operatorname{Ly} \alpha$ and higher Lyman series absorbers. When the Doppler parameter is unconstrained, we used mean $b=25$ $\mathrm{km} \mathrm{s}^{-1}$ to propagate uncertainties in the continuum slope of individual spectra.

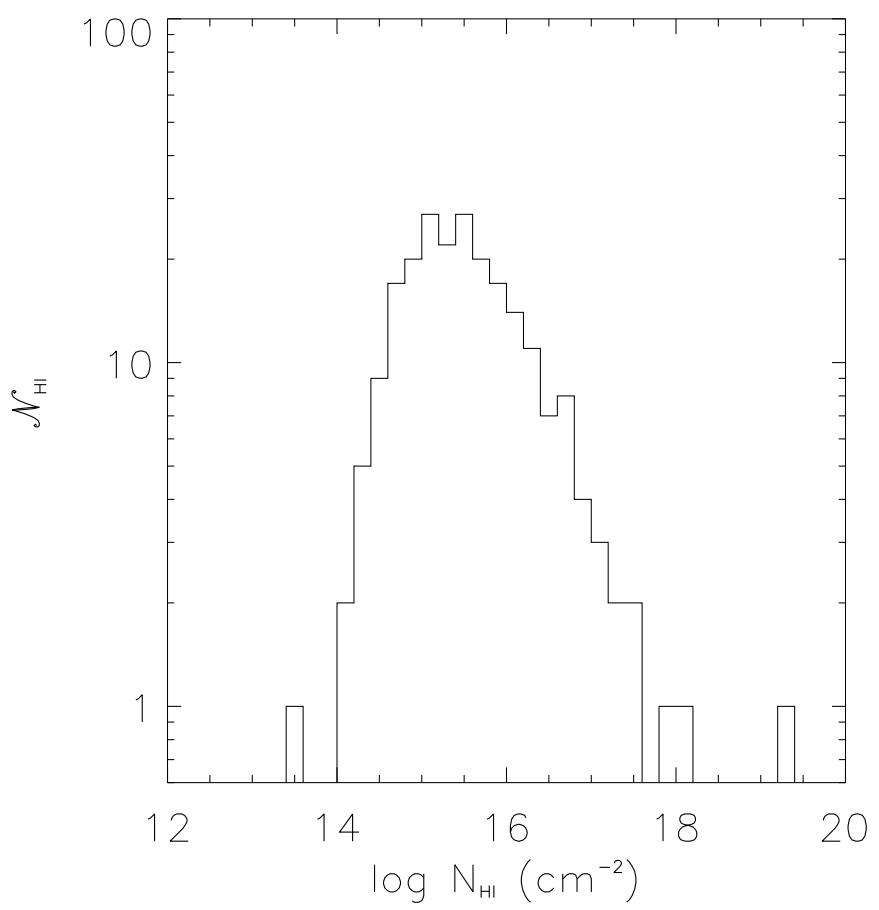

Figure 4. Distribution of strong $\mathrm{HI}_{\mathrm{I}}$ absorbers in column density $N_{\mathrm{HI}}$, with a range in absorber redshifts $\left(0.24326<z_{a}<0.91449\right)$ accessible to coverage with the COS moderate-resolution gratings (G130M and G160M). Along 71 AGN sight lines at $z_{\text {AGN }}>0.26$, we identified 214 pLLS with $14.5 \leqslant \log N_{\mathrm{H}_{\mathrm{I}}}<17.2$ and 7 LLS with $\log N_{\mathrm{H}_{\mathrm{I}}} \geqslant 17.2$. 
wavelength bin and is equal to the mean of the flux in the old pixels that overlap the new bin, weighted by the extent of overlap. The error arrays associated with the resampled spectra are determined using a weighting method similar to the flux rebinning. See Equations (2) and (3) in Telfer et al. (2002) for the rebinning formulae.

\section{OVERALL SAMPLE COMPOSITE SPECTRUM}

\subsection{Composite Construction}

To construct the overall composite spectrum we start by following the bootstrap method of Telfer et al. (2002) and then adapt the construction technique for our unique data set. To summarize the bootstrap technique, we start near the central region of the output composite spectrum, between $1050 \AA$ and $1150 \AA$, and normalize the spectra that include the entire central region to have an average flux value of one within the central region, which we refer to as the "central continuum window." We then include spectra in sorted order toward shorter wavelengths. Finally, we include the spectra in sorted order toward longer wavelengths. When a spectrum does not cover the central continuum window, we normalize it to the partially formed composite by finding the weightedmean normalization constant within multiple emission-line-free continuum windows, calculated using Equation (4) of Telfer et al. (2002). We form two independent composite spectra simultaneously: one of the fine-grained spectra showing the line-blanketing by the Ly $\alpha$ forest and interstellar absorption lines, and another of the spline fits to the individual spectra. The spline fits pass over the narrow absorption lines.

With our unique data set and spline fits, we adjust the composite construction method in five ways. First, with the identification in Paper I of broad emission lines from highly ionized species below $800 \AA$, we were able to restrict the normalization of the spectra at the highest redshifts to two narrow regions of continuum-like windows at $660-670 \AA$ and $715-735 \AA$. This is in contrast to using all of the flux, including that from emission lines below $800 \AA$ in the calculation of the normalization constant, as was done in our initial method. Our second adjustment also limits the normalization calculation to regions of continuum, which is our primary interest. We refine and narrow the continuum windows above $800 \AA$ to wavelengths $855-880 \AA$,

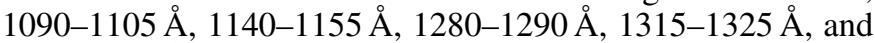
1440-1465 $\AA$. For our third adjustment, we choose the region between $855-880 \AA$ as the central continuum window because it is the largest of the narrowed EUV continuum windows with a large number of contributing spectra. Fourth, we note that the bootstrapping technique can be sensitive to the ordering in which one includes the spectra, especially at the beginning of the process when only a handful of spectra determine the shape of the composite. Therefore, we increase the number of spectra normalized at only the central continuum window from 40 to 70 by decreasing the required overlap with the central continuum window from $100 \%$ to $50 \%$. Last, because we are interested in characterizing the shape of the underlying continuum as a power law, we follow the approach of Vanden Berk et al. (2001) and combine the spectra as a geometric mean, which preserves power-law slopes. We also provide a mediancombined composite, which preserves the relative shape of the emission features.

Below $700 \AA$, the number of AGN spectra contributing to each $0.1 \AA$ bin in Figure 2 declines steadily. Several AGNs listed in Table 1 do not appear in this figure because their short wavelength spectra are masked out, owing to LLS absorption, airglow, and pLLS edges. The final overall sample composite spectra (both geometric-mean and median) are presented in two panels of Figure 5, covering rest-frame wavelengths from 475-1785 A. In each panel, we show both the fine-grained data with absorption lines included and the spline-fit continuum composites. In the geometric-mean spectrum, which we regard as the better characterization of the AGN composite, the effects of line-blanketing by the Ly $\alpha$ forest can be seen in the difference between the spline-fit composite and the real data composite. Figure 6 shows the optical depth, $\tau_{\lambda}$, arising from line-blanketing of the continuum by the Ly $\alpha$ forest at $\lambda<1150 \AA$. We derive optical depths from the difference in fluxes (red and black) in the geometric mean composite, shown in the top panel of Figure 5.

To characterize the continuum slope of the composite spectrum, we follow the simple approach of Vanden Berk et al. (2001). We calculate the slope between continuum regions of maximum separation on either side of the spectral break, which is clearly present in the composite around $1000 \AA$. Because the flux distribution, $F_{\lambda}$, flattens at shorter wavelengths, the two power-law fits pass under the observed spectrum and match at the break. To satisfy this condition, we first calculate the slope of a line connecting the minima of the two best continuum regions in $\log -\log$ space. We then divide the entire spectrum by this line, find the wavelengths of the minima, and calculate the slope of the line that connects the second pair of minima. This results in a line that does not cross the composite. We perform this calculation twice, once in the EUV and again in the FUV. We find a mean EUV spectral index $\alpha_{\lambda}=-0.59$ between line-free windows centered at $724.5 \AA$ and $859 \AA$, and mean FUV index $\alpha_{\lambda}=-1.17$ between $1101 \AA$ and $1449 \AA$. These wavelength indices correspond to frequency indices $\alpha_{v}$ of -1.41 (EUV) and -0.83 (FUV).

\subsection{Uncertainties}

We now discuss sources of random and systematic uncertainty in the composite spectral indices. As in Paper I, we fit two power laws to the spline composite spectrum, with indices $\alpha_{\mathrm{FUV}}$ and $\alpha_{\mathrm{EUV}}$, and match them at a break wavelength, which we find to be $\lambda_{\text {br }} \approx 1000 \AA$, consistent with Paper I and accurate to $\sim 50 \AA$. Although this gradual break is apparent in the composite, its presence is less clear in the individual spectra, owing to the limited spectral range of the COS observations and to strong emission lines of OVI $\lambda 1035$ and $\operatorname{Ly} \beta \lambda 1025$ near the break wavelength. Because the sample of AGNs in Paper I had no targets between $0.16<z<0.45$, most rest-frame spectra lay either blueward or redward of the $1000 \AA$ break. In our new sample, 55 AGNs have redshifts in that range, but we do not distinguish a clear break in individual spectra. With the limited wavelength coverage of COS (G130M, G160M), any single AGN spectrum does not have access to the four continuum windows needed to measure two distinct power laws that straddle the break.

To quantify the uncertainty in the fitting of the composite spectrum, we explore the sources of uncertainty described by Scott et al. (2004), including the effects of intrinsic variations in the shape of the SEDs, Galactic extinction, and formal statistical fitting errors. As in Paper I, we do not include the effects of intrinsic absorbers or interstellar lines, as these absorption lines are easily removed with our moderate-resolution COS spectra. However, we do consider the effects from the strongest systems in the Ly $\alpha$ forest. The largest source of uncertainty comes from the natural variations in the slope of the contributing spectra. 

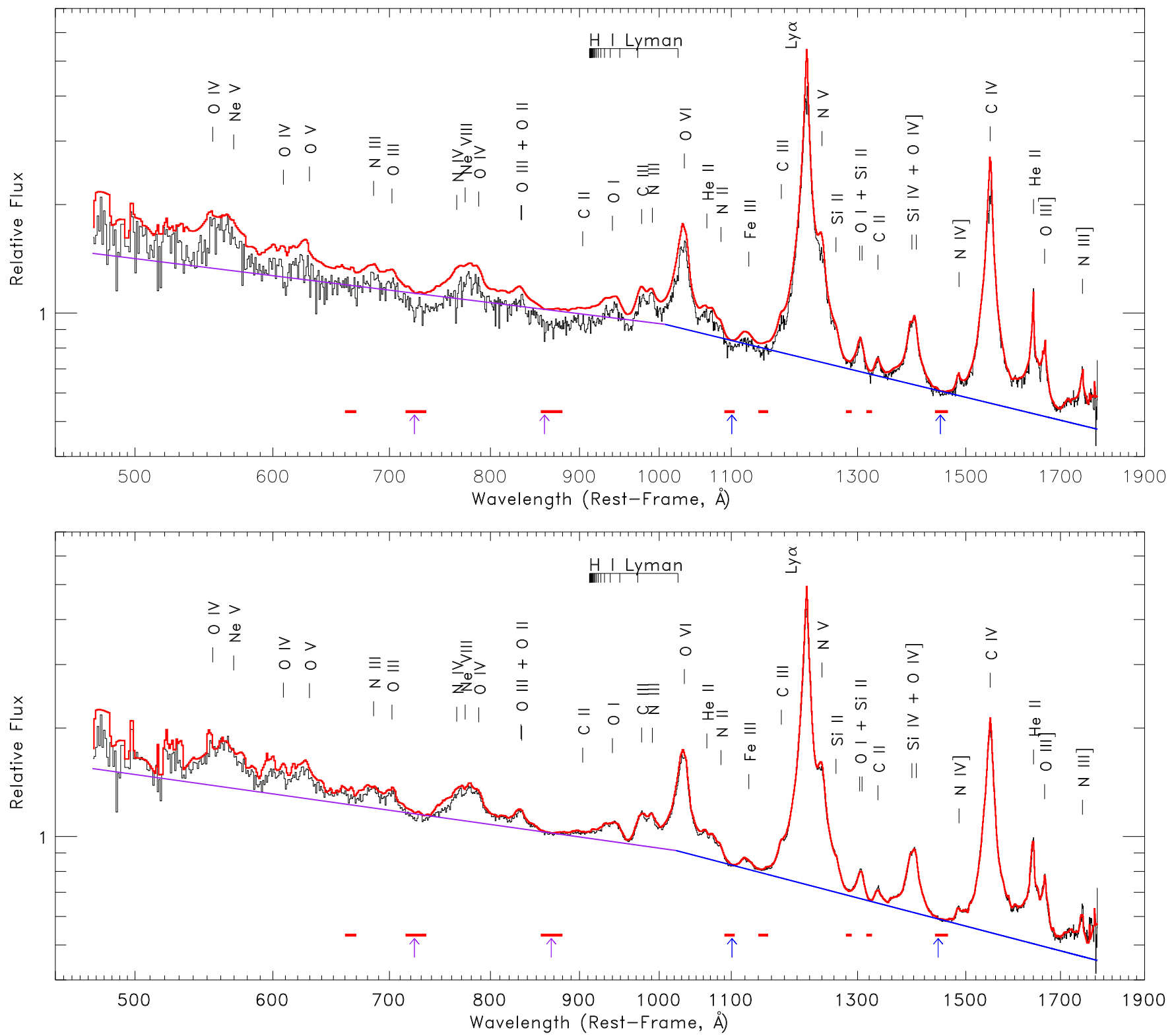

Figure 5. Composite spectra with rest-frame wavelengths $465-1750 \AA$ made from 159 AGNs with redshifts $0.001 \leqslant z_{\text {AGN }} \leqslant 1.476$, resampled to $0.1 \AA$, plotted in $1 \AA$ bins, normalized to unit flux at $1100 \AA$, and showing broad FUV and EUV emission lines atop a power-law continuum. Eight continuum windows are shown as small red boxes along the bottom. Composite data are shown in black; the red curve is a composite of individual spline fits. (Top) Geometric-mean-combined HST/COS spectrum with frequency distribution, $F_{v} \propto v^{\alpha_{\nu}}$, with break at $\lambda_{\text {br }} \approx 1000 \AA$ and spectral indices $\alpha_{v}=-1.41 \pm 0.15$ (EUV, $\lambda<1000 \AA$ ) and $\alpha_{v}=-0.83 \pm 0.09$ (FUV, $\lambda>1200 \AA$ ). (Bottom) Median-combined composite with break at $\lambda_{\mathrm{br}} \approx 1025 \AA$ and spectral indices $\alpha_{v}=-1.32 \pm 0.15$ (EUV) and $\alpha_{v}=-0.74 \pm 0.09$ (FUV).

(A color version of this figure is available in the online journal.)

We estimate this uncertainty by selecting 1000 bootstrap samples with replacement from our sample of 159 AGN spectra. The resulting distributions of spectral index in frequency lead to mean values: $\alpha_{\mathrm{EUV}}=-1.41 \pm 0.15$ and $\alpha_{\mathrm{FUV}}=-0.83 \pm 0.09$ in the EUV and FUV. Figure 7 shows a montage of spectra for individual AGNs, illustrating the wide range of spectral slopes and emission-line strengths.

We also investigated the range of uncertainties arising from UV extinction corrections from two quantities: $E(B-V)$ and $R_{V}$. We alter the measured $E(B-V)$ by $\pm 16 \%(1 \sigma)$ as reported by Schlegel et al. (1998). We deredden the individual spectra with $E(B-V)$ multiplied by 1.16 or 0.84 , compile the spectra into a composite, and fit the continua. Over these ranges, we find that the index $\alpha_{\mathrm{EUV}}$ changes by $(+0.064,-0.022)$ while $\alpha_{\mathrm{FUV}}$ changes by $(+0.046,-0.023)$. Next, we estimate the sensitivity to deviations from the canonical value $R_{V}=3.1$, which Clayton et al. (1988) found to vary from $R_{V}=2.5$ to $R_{V}=5.5$. We follow Scott et al. (2004) and deredden individual spectra with $R_{V}=2.8$ and $R_{V}=4.0$ and compiling the spectra into composites. We find that $\alpha_{\mathrm{EUV}}$ changes by $(+0.041,-0.051)$ and $\alpha_{\mathrm{FUV}}$ by $(+0.032,-0.059)$. We estimate the uncertainties arising from correcting pLLS absorption of strength $\log N_{\mathrm{HI}_{\mathrm{I}}} \geqslant 15.0$ by altering the measured column densities by $\pm 1 \sigma$ as reported in Table 2 . We find that $\alpha_{\mathrm{EUV}}$ changes by $(+0.037,-0.010)$ and $\alpha_{\mathrm{FUV}}$ by $(+0.011,-0.011)$. The formal statistical errors for the spectral indices are negligible $(<0.001)$ owing to the high $\mathrm{S} / \mathrm{N}$ 


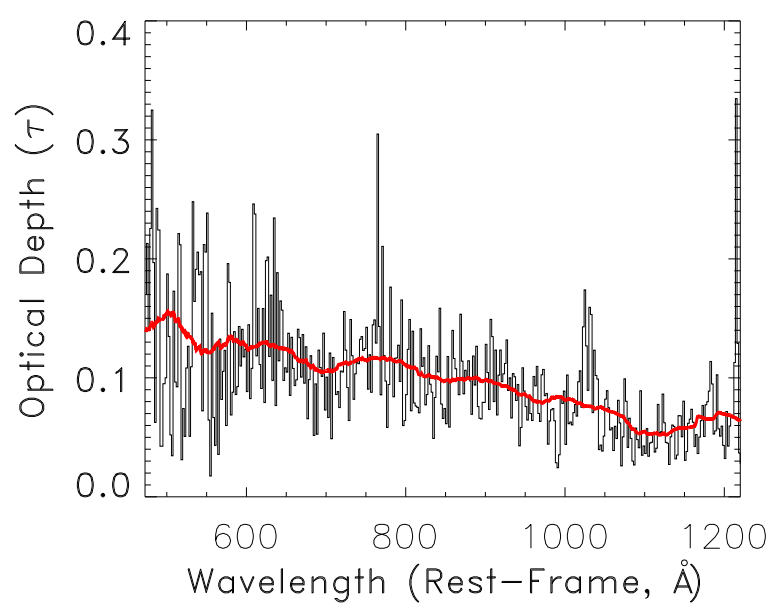

Figure 6. Optical depth arising from continuum line-blanketing by the Ly $\alpha$ forest, calculated from the difference in fluxes (top panel of Figure 5) between spline-fit continuum and line-blanketed data, and binned by 20 pixels of $0.1 \AA$ width. Red overplot shows optical depth, smoothed over 1000 pixels.

(A color version of this figure is available in the online journal.)

ratio of our composite spectra, therefore we do not include them in the final quoted uncertainties. We add the random uncertainties of cosmic variance with the systematic effects of correcting for extinction in quadrature and estimate the total uncertainties to be \pm 0.15 for $\alpha_{\mathrm{EUV}}$ and \pm 0.09 for $\alpha_{\mathrm{FUV}}$.

As noted earlier, we do not correct for AGN dust, but small amounts could be present as long as it does not produce a strong turnover in the far-UV fluxes. We can rule out a large amount of intrinsic extinction if it obeys a selective extinction law, $A(\lambda) / A_{V}$, that rises steeply at short (UV) wavelengths. Otherwise, we would see steep curvature in the rest-frame EUV rather than a power law.

\subsection{Comparison to Other Composite Spectra}

Ultraviolet spectra of AGNs have been surveyed by many previous telescopes, including the International Ultraviolet Explorer (O'Brien et al. 1988) and the HST Faint Object Spectrograph (Zheng et al. 1997). More recent AGN composite spectra were constructed from data taken with $H S T / F O S+S T I S$ (Telfer et al. 2002), FUSE (Scott et al. 2004), and HST/COS
(Paper I and this paper). Figure 8 compares the HST/COS composites with previous studies with $H S T / F O S+S T I S$ and FUSE. Our current COS survey finds essentially the same EUV spectral index, $\alpha_{v}=-1.41 \pm 0.15$, as found in Paper I, $\alpha_{v}=-1.41 \pm 0.22$, but with better statistics and coverage to shorter wavelengths (below $500 \AA$ ). This consistency is reassuring, as our current composite includes 159 AGN spectra, compared with 22 AGNs in the initial COS study (Paper I). The $H S T / C O S$ EUV index, $\alpha_{v} \approx-1.4$ is slightly harder than the HST/FOS+STIS value, $\alpha_{v}=-1.57 \pm 0.17$, found by Telfer et al. (2002) for 39 radio-quiet AGNs. However, both HST surveys found indices steeper than the FUSE slope, $\alpha_{v}=-0.56_{-0.28}^{+0.38}$ (Scott et al. 2004), a puzzling discrepancy that we now investigate.

The differences between continuum slopes found in FUSE and COS composite spectra are likely to arise from four general factors: (1) continuum placement beneath prominent EUV emission lines; (2) line blanketing by the Ly $\alpha$ forest and stronger (pLLS) absorbers; (3) continuum windows that span an intrinsically curved AGN spectrum; and (4) possible correlation of slope and AGN luminosity. High $\mathrm{S} / \mathrm{N}$ spectra at the moderate resolution of COS $(\mathrm{G} 130 \mathrm{M} / \mathrm{G} 160 \mathrm{M})$ are critical for identifying the underlying continuum near the strong EUV emission lines of O III, O IV, and O V and the Ne VIII doublet $(\lambda \lambda 770,780)$. The COS spectral resolution also allows us to fit over the narrow $\mathrm{H}$ I absorbers in the Ly $\alpha$ forest (factor number two) and restore the continuum absorbed by the stronger systems (LLS and pLLS). Factor three is a more subtle effect, but it may be the most important. The COS wavelength coverage (1135-1795 $\AA$ ) is broader than that of FUSE (912-1189 $\AA$ ), and it provides linefree continuum windows above and below $1100 \AA$, spanning an intrinsically curved SED. This allows us to construct a two-component spectrum with indices $\alpha_{v}=-1.41 \pm 0.15$ in the EUV (500-1000 $\AA$ ) and $\alpha_{v}=-0.83 \pm 0.09$ in the FUV (1200-2000 $\AA$ ) with a break at $\lambda_{\text {br }} \approx 1000 \pm 25 \AA$. Many of the COS sight lines observe higher-redshift AGNs that sample different regions of the SED than those of FUSE. Shortward of $912 \AA$, we place the continuum below a number of prominent emission lines, using nearly line-free continuum windows at $665 \pm 5 \AA, 725 \pm 10 \AA$, and $870 \pm 10 \AA$. Factor four refers to possible selection effects of AGN luminosity with redshift. Previous samples used targets at a variety of redshifts and luminosities, observed with different spectral resolution, FUV

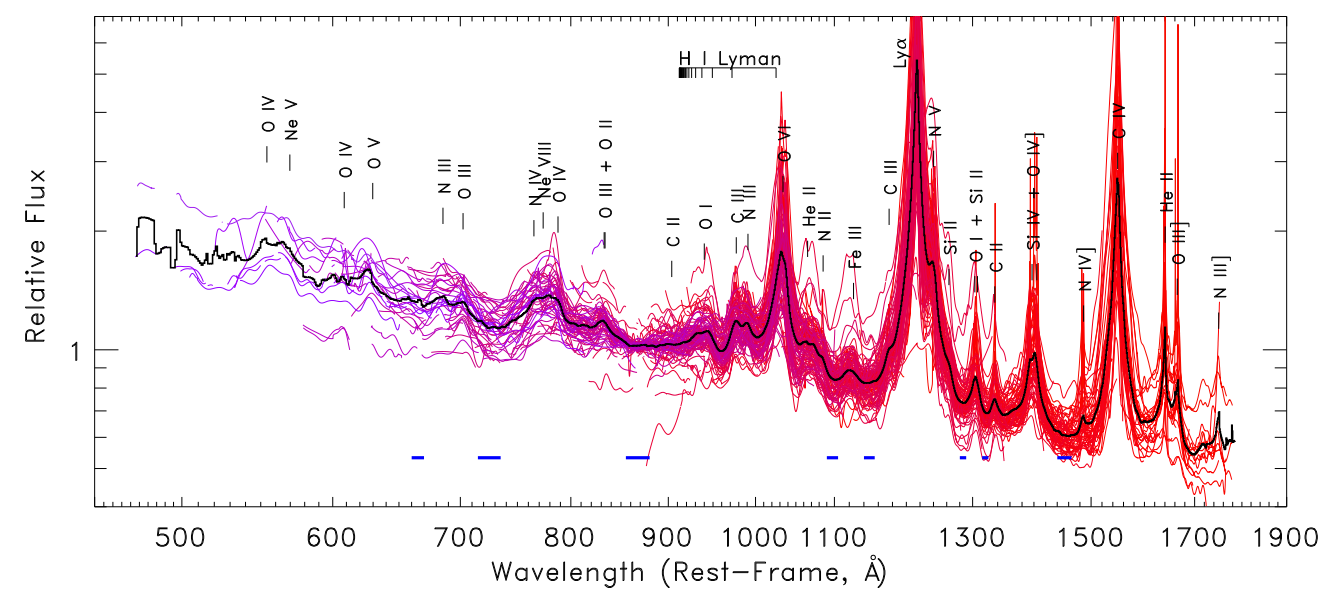

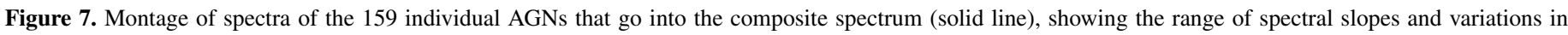
emission-line strengths. The line-free continuum windows are shown as blue bars along bottom.

(A color version of this figure is available in the online journal.) 

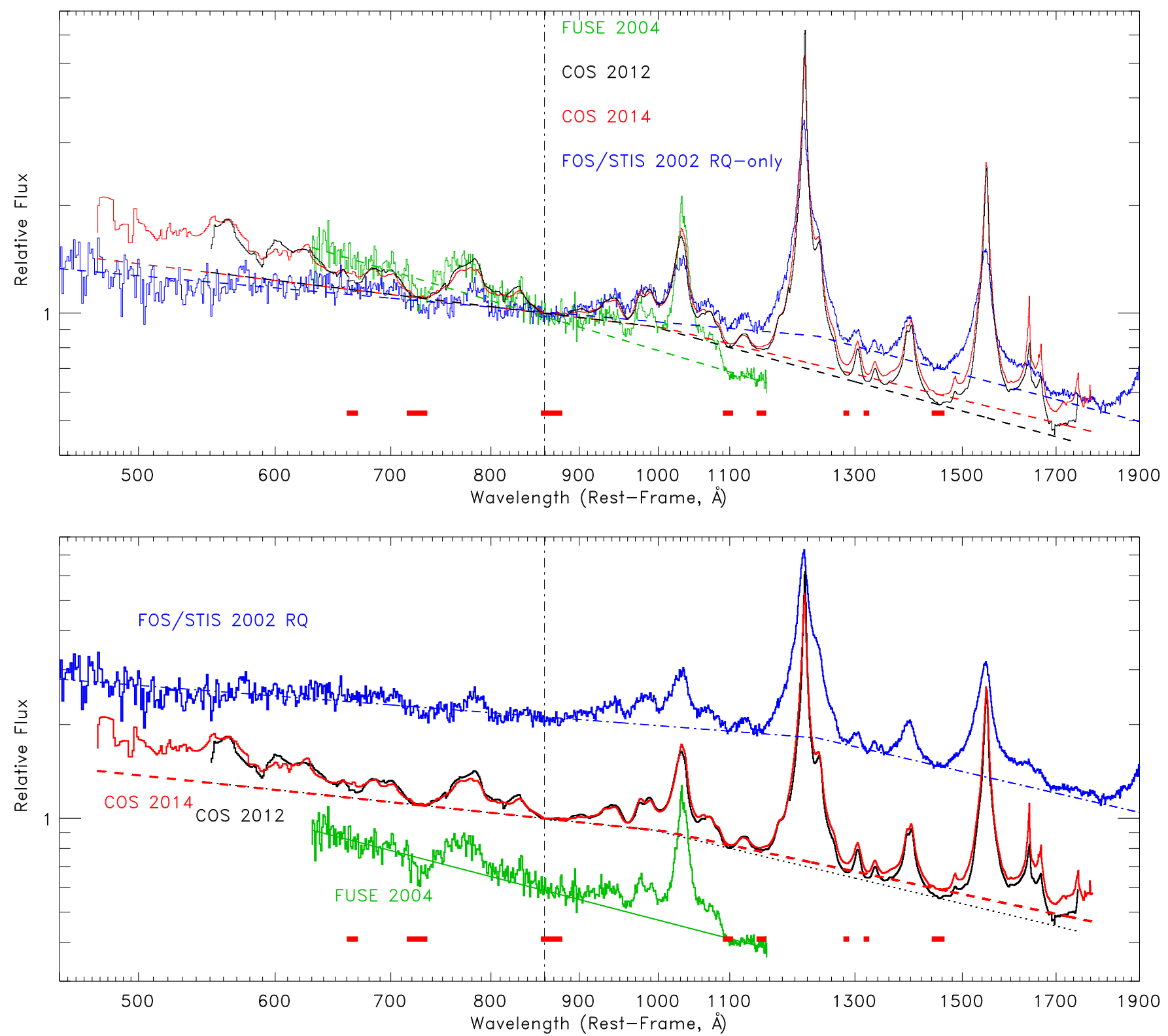

Figure 8. Comparison of the AGN composite spectra from FUSE (Scott et al. 2004), HST/FOS+STIS (Telfer et al. 2002), and two surveys with HST/COS (Paper I; this paper). Top: composite spectra, $F_{\lambda}$, all normalized at the $860 \AA$ line-free continuum window. Dashed lines show underlying power-law continua. The COS (2012, 2014) composite spectra, $F_{v} \propto v^{\alpha_{v}}$, have essentially the same EUV spectral index, $\alpha_{v}=-1.41 \pm 0.15$. FOS/STIS spectrum has $\alpha_{v}=-1.57 \pm 0.17$ for 39 radio-quiet QSOs. The FUSE spectrum is harder with $\alpha_{v}=-0.56_{-0.28}^{+0.38}$ based on lower-redshift AGNs. Bottom: spectra offset vertically for clarity. The different slopes arise from fitting the continuum beneath prominent broad EUV emission lines (Ne VIII, O III, O IV, O v) and $H S T / \mathrm{COS}$ wavelength coverage spanning an intrinsically curved AGN spectrum. Access to line-free continuum windows above and below $1100 \AA ̊$ (red bars along bottom) allows us to fit different FUV and EUV continuum slopes with a break at $\lambda_{\text {br }} \approx 1000 \pm 25 \AA$.

(A color version of this figure is available in the online journal.)

throughput, and instruments. All of the UV composite spectra (HST and FUSE) are based on the available UV-bright targets (Type 1 Seyferts and QSOs) studied with IUE and the Galaxy Evolution Explorer (GALEX). Most of these AGNs were chosen as background sources for studies of IGM, CGM, and Galactic halo gas. Although these targets are not a complete, flux-limited sample of the AGN luminosity function (e.g., Barger \& Cowie 2010), they probably are representative of UV-bright QSOs, at least at redshifts $z<0.4$.

Figure 9 compares the average AGN redshift per wavelength bin for the COS and FUSE surveys, overlaid on the linefree continuum windows. Evidently, the COS targets are at systematically higher redshift and their wavelength coverage is broader than that of FUSE. The average AGN luminosity also differs longward and shortward of the break. At $\lambda \approx$ $800 \AA$, the COS and FUSE composites are both probing similar luminosities. As shown in the top panel of Figure 9, the two spectral slopes are fairly similar between 650 and $1000 \AA$ and the only difference comes from the sudden decline in FUSE fluxes between 1090 and $1140 \AA$ A. Lacking the longerwavelength continuum windows, the FUSE spectra were unable to fit the break in spectral slope at longer wavelengths. Figure 10 shows the distributions of spectral index $\alpha_{\lambda}$ and the effects of the available continuum windows falling longward or shortward of the $1000 \AA$ break. The two-power-law fits possible with COS data allow us to measure the spectral curvature and distinguish 

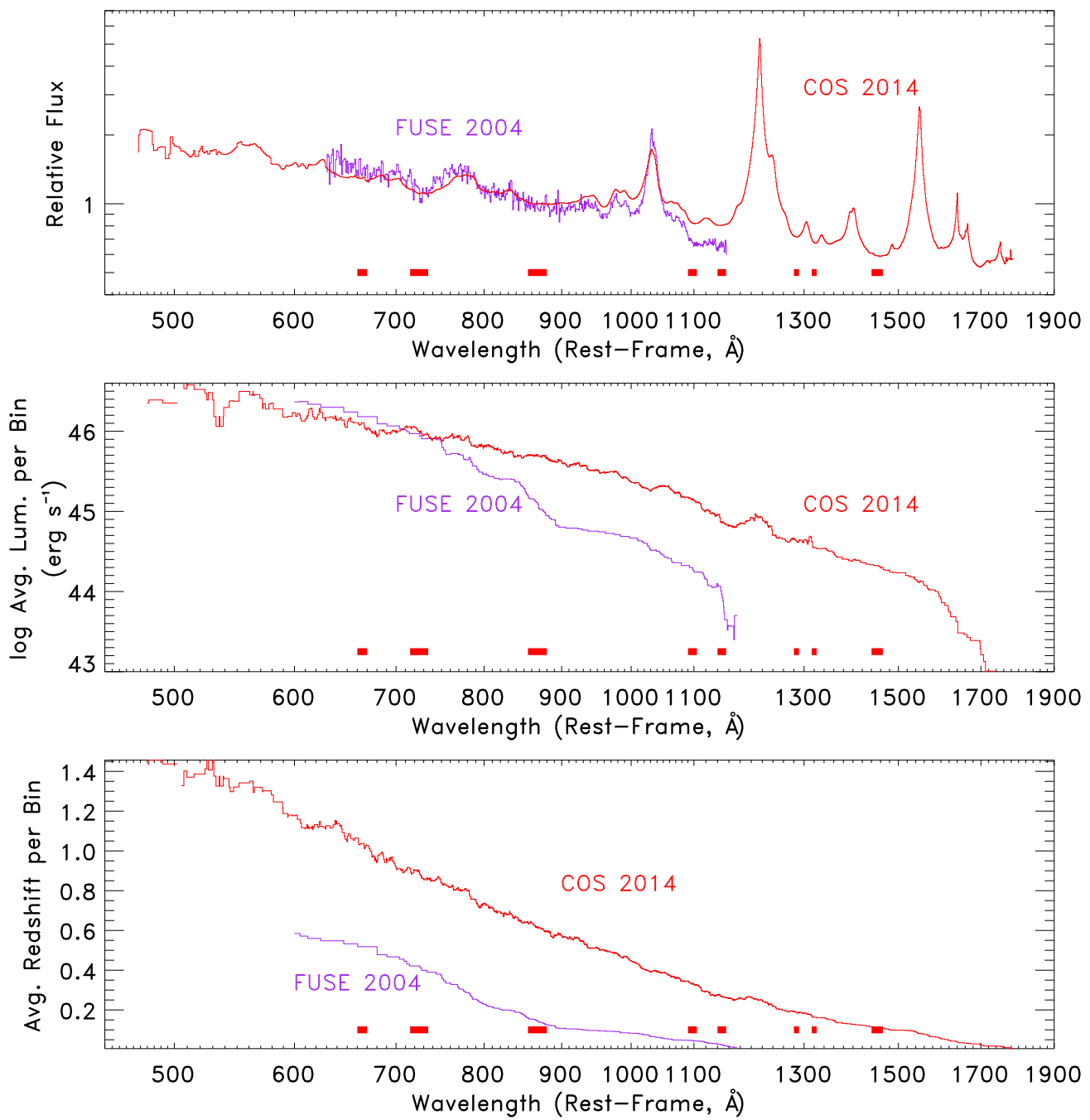

Figure 9. Comparison of composite spectra from COS and FUSE, with line-free continuum windows shown as red boxes at $660-670 \AA$, 720-730 $\mathrm{A}, 855-880 \AA$,

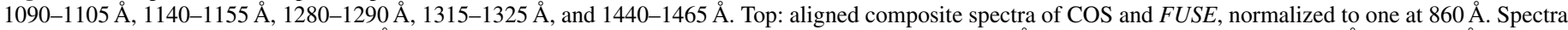
agree at short wavelengths $(\lambda \leqslant 1000 \AA)$ but the FUSE fluxes show a sudden dropoff longward of $1100 \AA$ in continuum windows at $1100 \AA$ and $1145 \AA$. Middle: average AGN luminosity per wavelength bin. Bottom: average redshift of AGNs per wavelength bin. We plot the geometric mean luminosity, $\lambda L_{\lambda}$, at $1000 \AA$. The FUSE survey samples AGNs at lower redshift than COS, with AGN luminosities comparable at $\lambda<800 \AA$, but much lower at $\lambda>900 \AA$.

(A color version of this figure is available in the online journal.)

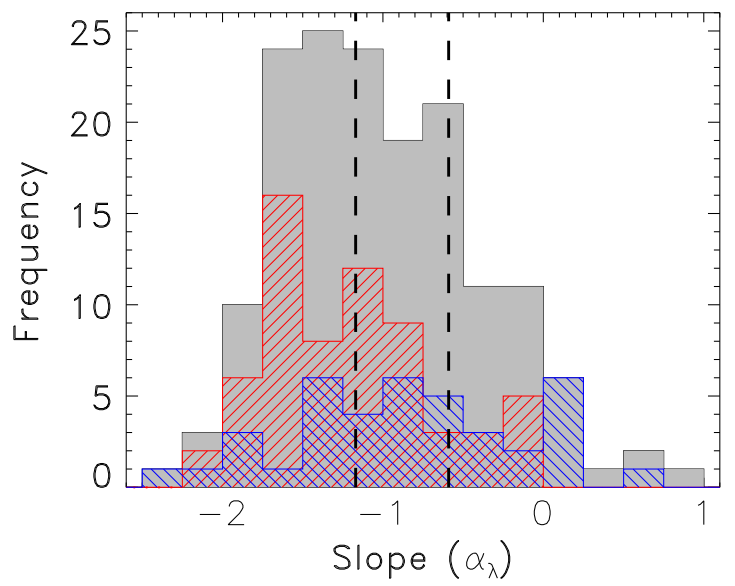

Figure 10. Distributions in spectral index, $\alpha_{\lambda}$, vs. redshift for $159 \mathrm{HST} / \mathrm{COS}$ AGNs, where $\left(\alpha_{\lambda}+\alpha_{v}\right)=-2$. Two vertical dashed lines mark mean values: $\left\langle\alpha_{\lambda}\right\rangle=-0.59$ for rest-frame EUV $(500-1000 \AA)$ and $\left\langle\alpha_{\lambda}\right\rangle=-1.17$ for rest-frame FUV (1200-2000 A). The red histogram shows spectra using two continuum windows redward of the $1000 \AA$ break; the blue histogram shows spectra using two continuum windows blueward of the break.

(A color version of this figure is available in the online journal.) between FUV and EUV slopes. This was not done with the FUSE composite fits.

In summary, we believe the $H S T / \mathrm{COS}$ composite spectra are superior owing to their higher spectral resolution $(\mathrm{G} 130 \mathrm{M}$ and G160M gratings) allowing us to resolve and mask out the Ly $\alpha$ forest and restore the continuum from stronger (LLS and pLLS) absorbers. The higher S/N of the COS spectra allow us to identify and resolve prominent UV/EUV emission lines and fit a more accurate underlying continuum. As shown in Figures 2 and 7 , the COS composite still contains fewer than 10 AGNs at $z>1$ that probe the rest-frame continuum at $\lambda<600 \AA$. These numbers are larger than in the earlier surveys, but the small sample means that the composite spectrum remains uncertain at the shortest wavelengths.

\subsection{Trends with Redshift, AGN Type, and Luminosity}

As in Paper I, we explore trends within the HST/COS AGN sample by constructing composite spectra based on various parameters and subsamples. Figure 11 shows the distributions of index $\alpha_{\lambda}$ in redshift, AGN activity type, Galactic foreground reddening, and monochromatic (1100 $)$ luminosity. In each 

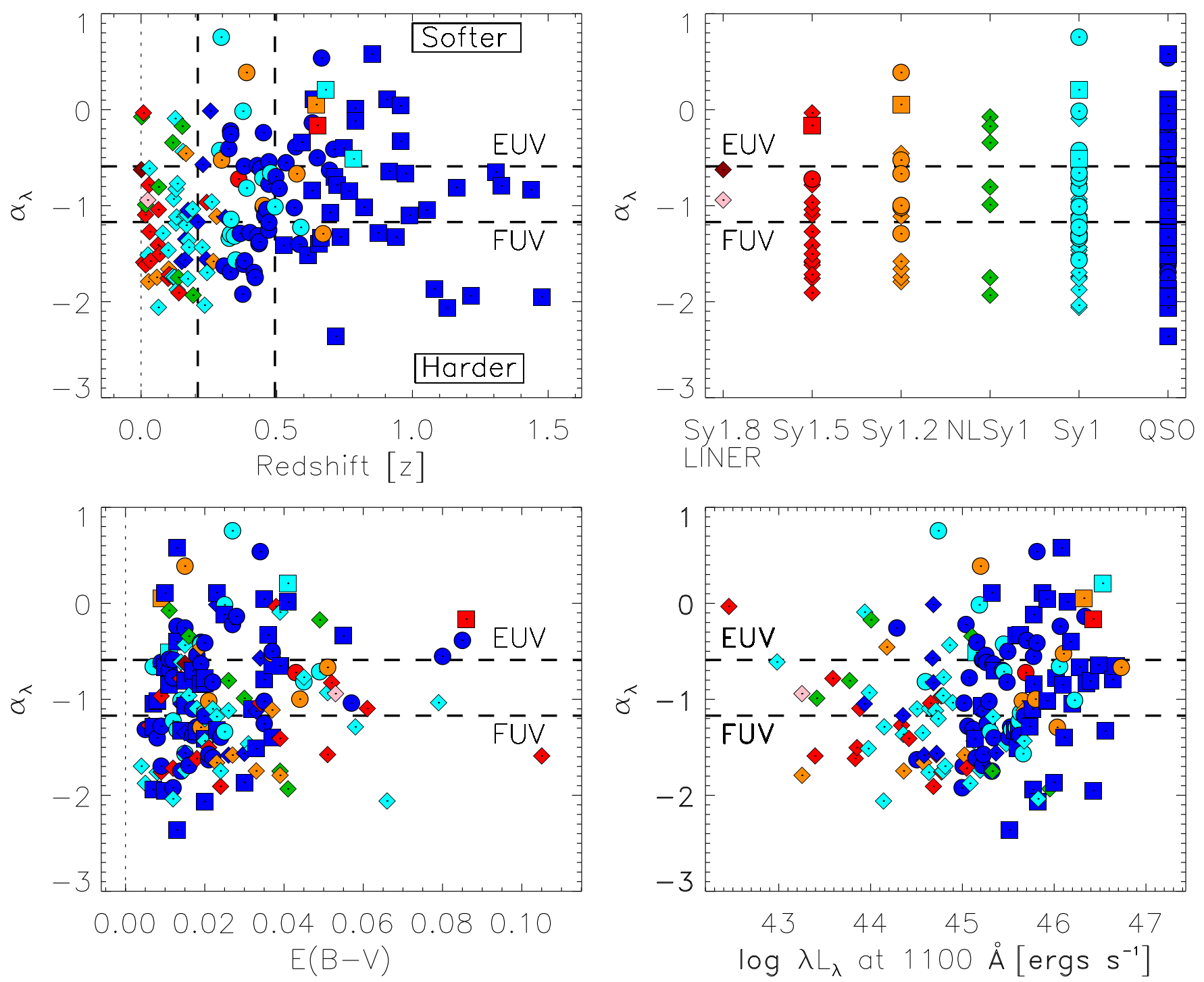

Figure 11. Top left: spectral index, $\alpha_{\lambda}$, vs. redshift for $159 \mathrm{HST} / \mathrm{COS}$ AGNs. Top right: index vs. activity type compiled by NED. Bottom left: index vs. $E(B-V)$. Bottom right: index vs. $1100 \AA$ monochromatic luminosity. Two low-luminosity AGNs (NGC 4395 and NGC 4051) with $\log \lambda L_{\lambda} \leqslant 41$ ) are omitted for clarity. Redshift and luminosity are correlated in these composites because of curvature in the underlying spectral shape. Two horizontal lines mark mean values of this index: $\left\langle\alpha_{\lambda}\right\rangle=-0.59$ for rest-frame EUV (500-1000 $)$; and $\left\langle\alpha_{\lambda}\right\rangle=-1.17$ for rest-frame FUV (1200-2000 $)$. Vertical lines mark boundaries between targets designated as low-redshift $(z<0.2)$, intermediate-redshift $(0.2<z<0.5)$, and high-redshift $(z>0.5)$. Colors denote AGN activity type: dark blue (QSO), cyan (Sy1), green (NLSy1), orange (Sy1.2), red (Sy1.5), pink (LINER), and brown (Sy1.8). Symbol shapes refer to windows used to fit continua: diamonds longward of 1000 A, squares shortward of $1000 \AA$, and circles straddling $1000 \AA$ with one window each in EUV and FUV.

(A color version of this figure is available in the online journal.)

panel, two horizontal lines denote the sample-mean values: $\left\langle\alpha_{\lambda}\right\rangle=-0.59$ for the rest-frame EUV (500-1000 $\AA$ ) band and $\left\langle\alpha_{\lambda}\right\rangle=-1.17$ for the rest-frame FUV (1200-2000 $\AA$ ) band. The spectral indices extend over a wide range of AGN luminosities and activity types with no obvious trend or correlation. Galactic reddening does not appear to produce any difference in the index. There may be subtle trends in the distribution of $\alpha_{\lambda}$ with redshifts because we are observing the rest-frame flux from an intrinsically curved SED. At low redshifts $(z<0.25)$, there are many AGNs with steep slopes, $\alpha_{\lambda}<-1.5$, indicating hard UV spectra. However, only seven AGNs have spectra with $\alpha_{\lambda}<-1.5$. At higher redshifts $(z>0.5)$, there are few AGNs with slopes $\alpha_{\lambda}<-1.5$, and the survey contains few AGNs at the most extreme redshifts $(z>1)$. Many more have soft spectra with slopes $\alpha_{\lambda}>-0.5$.

\subsection{Softened UV Spectra and Continuum Edges}

Accretion disk (AD) model spectra have recently been investigated by a number of groups (Davis et al. 2007; Davis \& Laor 2011; Done et al. 2012; Slone \& Netzer 2012) with a goal of comparing to UV and EUV spectra. The observed farUV spectral turnover at $\lambda<1000 \AA$ limits the maximal disk temperature to $T_{\max } \approx 50,000 \mathrm{~K}$. Model atmospheres computed with the TLUSTY code (Hubeny et al. 2001) and including winds driven from inner regions of the disk predict a spectral break near $1000 \AA$, arising from the Lyman edge $(912 \AA)$ and wind-truncation of the hot inner part of the disk (Laor \& Davis 2014). In a standard multi-temperature accretion disk models with blackbody spectra in annular rings (Pringle 1981), the radial temperature distribution scales as $T(r) \propto\left(M_{\mathrm{BH}} M / r^{3}\right)^{1 / 4}$. 
In their models of wind-ejecting disks, Slone \& Netzer (2012) suggest that the spectral shape is governed by the radial profile of $\dot{M}(r)$, and the radius $r_{1 / 2}$ where half the disk mass has been ejected. Their observational predictions are based on the removal of hot accreting gas from the inner regions of the AD and accompanying removal of energy from the UV-emitting portions of the SED. A large mass accretion rate throughout the AD produces higher luminosities and shifts the SED to shorter (UV) wavelengths. The closer $r_{1 / 2}$ comes to the innermost stable circular orbit, $r_{\mathrm{ISCO}}$, the more FUV and EUV radiation will be emitted.

Slone \& Netzer (2012) used the sensitivity of the EUV spectral index, $\alpha_{456-912}$ between 456 and $912 \AA$, to constrain accretion properties outside $R_{\mathrm{ISCO}}$, the radius of the innermost stable circular orbit around a black hole (see their Figure 7). Their model is governed by mass accretion rates, $\dot{M}_{\text {in }}$ and $\dot{M}_{\text {out }}$, at the inner and outer disk radii of the disk, relative to the Eddington accretion rate $\dot{M}_{\text {edd }}$ and luminosity $L / L_{\text {edd }}$ relative to the Eddington luminosity, $L_{\text {edd }}$. From the observed EUV spectral index, $\alpha_{456-912} \approx-1.4$, we constrain the mean AGN accretion rate and luminosity to values $\dot{M}_{\text {in }} / \dot{M}_{\text {edd }}<0.1$ and $L / L_{\text {edd }}<0.2$. We caution that these inferences are subject to the validity of accretion disk model atmospheres, including effects of external irradiation, uncertainty in where energy is being deposited, and the role of magnetic field energy dissipation. In addition, disk photospheres may differ from those of hot stars, with spatially variable $\tau=1$ surfaces.

Laor \& Davis (2014) explore similar disk-truncation models, solving for the radial structure of a disk with mass loss. They find that the wind mass loss rate, $\dot{M}_{\text {wind }}$, becomes comparable to the total accretion rate $\dot{M}$ at radii a few tens of gravitational radii, $\left(G M / c^{2}\right)$. Line-driven winds set a cap of $T_{\max }<10^{5} \mathrm{~K}$ on their disks, which in most cases are truncated well outside the ISCO radius. These models are consistent with the observed SED turnover at $\lambda<1000 \AA$ that is weakly dependent on luminosity $L$ and black hole mass $M_{\mathrm{BH}}$. Their models of line-driven winds also cap $\mathrm{AD}$ effective temperatures, $T_{\text {eff }}<10^{5} \mathrm{~K}$. The UV spectral turnover is produced by both an $\mathrm{H}$ i Lyman edge and the limit on disk temperature.

Standard models of accretion disk atmospheres are predicted to exhibit $\mathrm{H}$ I and He I continuum edges at $912 \AA$ and $504 \AA$, respectively. This issue and the EUV (soft X-ray) spectra of accretion disks have been discussed by many authors (e.g., Kolykhalov \& Sunyaev 1984; Koratkar \& Blaes 1999; Done et al. 2012). The absence of any continuum absorption at $912 \AA$ in the composite spectrum was noted in Paper I, where we set an optical depth limit of $\tau_{\mathrm{H}_{\mathrm{I}}}<0.03$. From the 159 AGN composite (see Figures 5 and 8), our limit is now $\tau_{\mathrm{H}_{\mathrm{I}}}<0.01$ derived from the flux around $914.5 \AA$ and $910.5 \AA$. The limit for the He I edge at $504 \AA$ is less certain because of the difficulty in fitting the local continuum under neighboring broad EUV emission lines. However, from the general continuum shape between 480 and $520 \AA$, we can limit the He I continuum optical depth to $\tau_{\text {HeI }}<0.1$. Additional COS/G140L data now being acquired toward 11 AGNs at redshifts $1.5 \leqslant z \leqslant 2.2$ probe the rest-frame continua at $\lambda<400 \AA$ with good spectral coverage at the $504 \AA$ edge. We continue to see no He I continuum edge.

\section{DISCUSSION AND CONCLUSIONS}

We now summarize the results and implications of our HST/ COS survey of AGN spectral distributions in the AGN restframe FUV and EUV. Using spectra of 159 AGNs taken with HST/COS G130M and G160M gratings, we constructed a two- component composite spectrum in the EUV (500-1000 $)$ ) and FUV (1200-2000 ̊). These two spectral fits match at a break wavelength $\lambda_{\text {br }} \approx 1000 \AA$, below which the SED steepens to $F_{v} \propto v^{-1.41}$. The EUV index is the same as found in Paper I, but with smaller error bars. It is slightly harder than the index, $\alpha_{v}=-1.57 \pm 0.17$, found from the HST/FOS+STIS survey (Telfer et al. 2002) for radio-quiet AGNs, but much softer than the index, $\alpha_{v}=-0.56_{-0.28}^{+0.38}$, from the FUSE survey (Scott et al. 2004). These composite spectra are based on small numbers of AGNs with redshifts $(z \geqslant 1)$ sufficient to probe below $600 \AA$. However, the HST/COS survey provides a superior measure of the true underlying continuum. Our G130M/G160M data have sufficient spectral resolution and $\mathrm{S} / \mathrm{N}$ to mask out narrow lines from the $\operatorname{Ly} \alpha$ forest and restore the continuum from stronger (LLS and pLLS) absorbers. We also fit the continuum below the prominent broad EUV emission lines using nearly line-free continuum windows at $665 \pm 5 \AA, 725 \pm 10 \AA$, and $870 \pm 10 \AA$.

Our primary conclusions are as follows.

1. The HST/COS composite spectrum follows a flux distribution with $F_{v} \propto v^{-0.83 \pm 0.09}$ for AGN rest-frame wavelengths $1200-2000 \AA$ and $F_{v} \propto v^{-1.41 \pm 0.15}$ for $500-1000 \AA$. This EUV spectral index is slightly harder than that used in recent simulations (Haardt \& Madau 2012) of IGM photoionization and photoelectric heating.

2. Individual spectra of the 159 AGNs surveyed exhibit a wide range of spectral indices in the EUV, with typical values between $-2 \leqslant \alpha_{v} \leqslant 0$. These indices are local slopes and not characteristic of the SED over the full UV/EUV band.

3. The composite SED exhibits a turnover at $\lambda<1000 \AA$, characteristic of accretion disk models in which the maximum temperature $T_{\max }<10^{5} \mathrm{~K}$ and the inner disk is truncated by line-driven winds.

4. We see no continuum edges of H I (912 $\AA$ ) or He I (504 $\AA$ ), with optical depth limits $\tau_{\mathrm{H}}<0.01$ and $\tau_{\mathrm{HeI}}<0.1$. The absence of these edges suggests that accretion disk atmospheres differ from those of hot stars because of external irradiation or inverted temperature structures arising from magnetic energy dissipation.

5. We find no obvious correlations of the EUV spectral index with interstellar reddening, AGN type, redshift, or luminosity $\left(\lambda L_{\lambda}\right.$ at $\left.1100 \AA\right)$. Such trends are difficult to pick out because the observable HST/COS (G130M/G160M) wavelength band (1135-1795 $\AA$ ) covers different portions of the SED over the AGN redshifts $(0.001<z<1.476)$ in our sample. The quoted indices, $\alpha_{\lambda}$, are local slopes that fall either in the FUV or EUV depending on AGN redshift.

6. The mean EUV slopes, compared to models of windtruncated thin accretion disks, constrain the mean accretion rate in the inner disk and the AGN luminosity to values $\dot{M}_{\text {in }} / \dot{M}_{\text {edd }}<0.1$ and $L / L_{\text {edd }}<0.2$ relative to their Eddington rates.

The order of magnitude improvement in sensitivity offered by COS over previous spectrographs has greatly increased the number of targets available for moderate-resolution UV spectroscopy. Some of these spectra have $\mathrm{S} / \mathrm{N}$ below the threshold chosen for this survey, and many are low-resolution (G140L) rather than G130M/G160M used here. Nevertheless, some of these archival spectra will provide EUV coverage down to $500 \AA$ (with $\sim 40 \mathrm{AGNs}$ ) and to $912 \AA$ (with $\sim 100 \mathrm{AGNs}$ ). Additional data at AGN rest wavelengths 400-500 $\AA$ would be helpful in fitting the SED deeper into the EUV, where fewer than 10 AGN sight lines have been probed to date with 
COS/G130M. Currently, our composite spectrum includes 10 AGNs that contribute at $\lambda \leqslant 600 \AA$ but only two AGNs at $\lambda<500 \AA$. As noted in Paper I, one can explore even shorter rest-frame wavelengths (304-500 $\AA$ ) using the sample of "He II quasars" (Worseck et al. 2011; Syphers et al. 2011; Shull et al. 2010) that probe the He II epoch of reionization at $z \approx 2.5-3.5$. In Hubble Cycle 21, we are observing 11 new AGN targets at $z=1.45$ to $z=2.13$, using the lower-resolution (G140L) grating. The first 10 of these spectra have now been acquired. After reduction, they should improve the accuracy of the composite spectrum down to $400 \AA$ and provide more sight lines that cover the He I $504 \AA$ continuum edge. Our intent is to create a composite AGN spectrum between $350 \AA$ and $1800 \AA$ using HST/COS archival spectra of AGNs with a variety of types and luminosities.

We thank the COS/GTO team for help on the calibration and verification of COS data. We acknowledge helpful discussions with Shane Davis, Ari Laor, and Jim Pringle on accretion disk models. We also thank the referee for helpful comments that encouraged us to explore the differences between COS and FUSE composites. This research was supported by NASA grants NNX08-AC14G and NAS5-98043 and the Astrophysical Theory Program (NNX07-AG77G from NASA) at the University of Colorado Boulder. J.M.S. thanks the Institute of Astronomy, Cambridge University, for their stimulating scientific atmosphere and support through the Sackler Visitor program.

\section{REFERENCES}

Barger, A. J., \& Cowie, L. L. 2010, ApJ, 718, 1235

Clayton, G. C., Cardelli, J. A., \& Mathis, J. S. 1988, ApJL, 329, L33
Danforth, C. W., Tilton, E. M., Shull, J. M., et al. 2014, ApJ, submitted (arXiv:1402.2655)

Davis, S. W., \& Laor, A. 2011, ApJ, 728, 98

Davis, S. W., Woo, J.-H., \& Blaes, O. M. 2007, ApJ, 668, 682

Done, C., Davis, S. W., Jin, C., Blaes, O., \& Ward, M. 2012, MNRAS, 420, 1848

Finn, C. W., Morris, S. M., Crighton, N. H. M., et al. 2014, MNRAS, 440,3317

Fitzpatrick, E. L. 1999, PASP, 111, 63

Gil de Paz, A., \& Madore, B. F. 2005, ApJ, 156, 345

Green, J. C., Froning, C., Osterman, S., et al. 2012, ApJ, 744, 60

Haardt, F., \& Madau, P. 2012, ApJ, 746, 125

Hubeny, I., Blaes, O., Krolike, J. H., \& Agol, E. 2001, ApJ, 559, 680

Kolykhalov, P. I., \& Sunyaev, R. A. 1984, AdSpR, 3, 249

Komatsu, E., Smith, K. M., Dunkley, J., et al. 2011, ApJS, 192, 18

Koratkar, A., \& Blaes, O. 1999, PASP, 111, 1

Krolik, J. H. 1999, Active Galactic Nuclei (Princeton, NJ: Princeton Univ. Press) Laor, A., \& Davis, S. W. 2014, MNRAS, 438, 3024

O'Brien, P. T., Wilson, R., \& Gondalekhar, P. 1988, MNRAS, 233,801

Osterman, S., Green, J., Froning, C., et al. 2011, Ap\&SS, 335, 257

Pringle, J. E. 1981, ARA\&A, 19, 137

Schlafly, E. F., \& Finkbeiner, D. P. 2011, ApJ, 737, 103

Schlegel, D. J., Finkbeiner, D. P., \& Davis, M. 1998, ApJ, 500, 525

Scott, J. E., Kriss, G. A., Brotherton, M., et al. 2004, ApJ, 615, 135

Shull, J. M., France, K., Danforth, C. W., Smith, B. D., \& Tumlinson, J. 2010, ApJ, 722, 1312

Shull, J. M., Stevans, M., \& Danforth, C. W. 2012, ApJ, 752, 152 (Paper I)

Slone, O., \& Netzer, H. 2012, MNRAS, 426, 656

Syphers, D., Anderson, S. F., Zheng, W., et al. 2011, ApJ, 726, 111

Telfer, R., Zheng, W., Kriss, G. A., \& Davidsen, A. F. 2002, ApJ, 565,773

Vanden Berk, D. E., Richards, G. T., Bauer, A., et al. 2001, AJ, 122, 549

Worseck, G., Prochaska, J. X., McQuinn, M., et al. 2011, ApJL, 733, L24

Zheng, W., Kriss, G. A., Telfer, R. C., Grimes, J. P., \& Davidsen, A. F. 1997, ApJ, 475,469 